\title{
Underwater Wireless Sensor Network's Performance Enhancement with Cooperative Routing and Sink Mobility
}

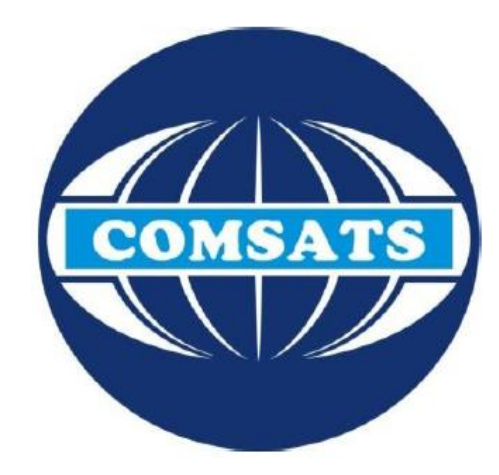

\author{
By \\ Amara Umar \\ CIIT/FA12-REE-012/ISB \\ MS Thesis \\ In \\ Electrical Engineering \\ COMSATS Institute of Information Technology \\ Islamabad - Pakistan
}

Fall, 2014 


\title{
Underwater Wireless Sensor Network's Performance Enhancement with Cooperative Routing and Sink Mobility
}

\author{
A Thesis Presented to \\ COMSATS Institute of Information Technology, Islamabad \\ In partial fulfillment \\ of the requirement for the degree of \\ MS (Electrical Engineering) \\ By \\ Amara Umar \\ CIIT/FA12-REE-012/ISB
}

Fall, 2014 


\section{Underwater Wireless Sensor Network's Performance Enhancement with Cooperative Routing and Sink Mobility}

A Post Graduate Thesis submitted to the Department of Electrical Engineering as partial fulfillment of the requirement for the award of Degree of M.S (Electrical Engineering).

\begin{tabular}{|c|c|}
\hline Name & Registration Number \\
\hline Amara Umar & CIIT/FA12-REE-012/ISB \\
\hline
\end{tabular}

\section{Supervisor}

Dr. Nadeem Javaid, Assistant Professor, Center for Advanced Studies in Telecommunications (CAST), COMSATS Institute of Information Technology (CIIT), Islamabad Campus,

November, 2014. 
Final Approval

This thesis titled

\title{
Underwater Wireless Sensor Network's Performance Enhancement with Cooperative Routing and Sink Mobility
}

\author{
By \\ Amara Umar \\ CIIT/FA12-REE-012/ISB
}

has been approved

For the COMSATS Institute of Information Technology, Islamabad

External Examiner:

Dr. Muhammad Sher

Professor, Department of Computer Science \& Software Engineering

Faculty of Basic and Applied Sciences

IIU, Islamabad

Supervisor:

Dr. Nadeem Javaid

Assistant Professor, Center for Advanced Studies in Telecommunications (CAST)

CIIT, Islamabad

HoD:

Dr. Shahid A. Khan

Professor, Department of Electrical Engineering

CIIT, Islamabad 


\section{Declaration}

I Amara Umar, CIIT/FA12-REE-012/ISB hereby declare that I have produced the work presented in this thesis, during the scheduled period of study. I also declare that I have not taken any material from any source except referred to wherever due that amount of plagiarism is within acceptable range. If a violation of HEC rules on research has occurred in this thesis, I shall be liable to punishable action under the plagiarism rules of the HEC.

Signature of the student:

Date:

Amara Umar

CIIT/FA12-REE-012/ISB 


\section{Certificate}

It is certified that Amara Umar, CIIT/FA12-REE-012/ISB has carried out all the work related to this thesis under my supervision at the Department of Electrical Engineering, COMSATS Institute of Information Technology, Islamabad and the work fulfills the requirements for the award of the MS degree.

Date:

Supervisor:

Dr. Nadeem Javaid

Assistant Professor

Head of Department:

Dr. Shahid A. Khan

Professor, Department of Electrical Engineering 


\section{DEDICATION}

\section{This thesis is dedicated to my family.}


vii

\section{ACKNOWLEDGMENT}

I am heartily grateful to my supervisor, Dr. Nadeem Javaid, whose patience, encouragement, guidance and insightful criticism from the beginning to the final level enabled me to have a deep understanding of the thesis. I would like to thank for his support throughout my degree keeping me going when times were tough, asking insightful questions, and offering invaluable advice.

Lastly, I offer my profound regard and blessing to everyone who supported me in any respect during the completion of my thesis, especially my husband in every way offered much assistance before, during, and at the completion stage of this thesis work.

Amara Umar CIIT/FA12-REE-012/ISB 
viii

\section{ABSTRACT \\ Underwater Wireless Sensor Network's Performance Enhancement with Cooperative Routing and Sink Mobility}

Underwater Wireless Sensor Networks (UWSNs) have attracted much concentration to support various applications like pollution monitoring, tsunami warnings, offshore exploration, tactical surveillance, etc. Network's efficiency and reliability in terms of high throughput, energy conservation, low Bit Error Rate (BER) and reduced delay are pre-requisites for many applications. However, distinctive features of UWSNs like low available bandwidth, large propagation delay, highly dynamic network topology, and high error probability pose many challenges for devising efficient and reliable communication protocols. In this thesis, we therefore propose a protocol along with its mathematical model that focuses on enhancing network reliability and efficiency via cooperative routing and sink mobility. Cooperative transmission is well known for reliable data transfer. Many cooperative communication protocols have been developed which investigate the physical and MAC layer aspects to improve link efficiency in harsh underwater environment, however, at network layer, it is still unexplored. Similarly, cooperative routing is not yet collaborated with sink mobility. We present a dominating set based cooperative routing algorithm at network layer. In this algorithm, potential relay and destination nodes for cooperative routing are selected on the basis of their depth and residual energy information. Data from source node is forwarded towards the destination node via relay node in a cooperative manner. Mobile Sinks directly gather data from destination nodes. Two different partner node selection criteria are also implemented and compared in this work. Selection of partner node (relay/destination node) for cooperative routing is to be performed on the basis of a certain criterion so that effective results can be achieved. We consider source node's depth threshold, potential relay/destination nodes's residual energy and Signal to Noise Ratio of the link connecting source node with potential relay/destination node as selection parameters. Based on the comprehensive simulations implemented in MATLAB, we observe that our proposed work improves the performance in terms of network lifetime, energy efficiency, throughput and reduces BER as compared to the existing depth based routing protocols. 


\section{$\underline{\text { List of Publications }}$}

1.: A. Umar, M. A. Hasnat, M. Behzad, I. Baseer, Z. A. Khan, U. Qasim, N. Javaid "On Enhancing Network Reliability and Throughput for Critical-range based Applications in UWSNs," accepted in The $9^{\text {th }}$ International Conference on Future Networks and Communications(FNC'14), 2014.

2.: A. Umar, M. Akbar, S. Ahmed, N. Javaid, Z. A. Khan, U. Qasim, " Underwater Wireless Sensor Network's Performance Enhancement with Cooperative Routing and Sink Mobility," accepted in The 9th IEEE International Conference on Broadband and Wireless Computing, Communication and Applications (BWCCA'14), 2014.

3.:Y. Moeen, N. Javaid, F. Saleem, A. Umar, M. Akbar, Z. A. Khan, U. Qasim, " MASEHA: Multi-hop Communication Using Active and Sleep Nodes For Energy Hole Avoidance in Wireless Sensor Networks," accepted in The 9th IEEE International Conference on Broadband and Wireless Computing, Communication and Applications (BWCCA'14), 2014

4. A. Umar, and N. Javaid, "Partner Node Selection Criteria for Cooperative Routing in Underwater WSNs," $5^{\text {th }}$ National Symposium on Information Technology: Towards Smart World (NSITNSW'15), Riyadh, KSA, 2015. 


\section{TABLE OF CONTENTS}

1 Introduction $\quad 1$

2 Literature Review $\quad 4$

3 On Enhancing Network Reliability and Throughput for Critical-Range Based Applications in UWSNs $\quad 7$

3.1 Motivation and contribution . . . . . . . . . . . . 8

3.2 Proposed Scheme . . . . . . . . . . . . . . . . . . . . . . . 8

3.2.1 Network Architecture . . . . . . . . . . . . . . . 8

3.2.2 Underwater Channel Model . . . . . . . . . . . . . . . . . 9

3.2.3 Network Initialization Phase . . . . . . . . . . . . . . . 10

3.2.4 Critical-range Based Data Sensing . . . . . . . . . . . . . . 10

3.2.5 Data Routing Phase . . . . . . . . . . . . . . . . . . 11

3.2.5.1 Depth Threshold Selection Process . . . . . . . . . . 12

3.2.5.2 Mobility Pattern of Courier Nodes . . . . . . . . . . . 13

3.2.5.3 Holding Time Computation on the Basis of Forwarding Function ................... 13

3.3 Simulation Results . . . . . . . . . . . . . . . . . . . . 15

4 Partner Node Selection Criteria for Cooperative Routing in UWSN 19

4.1 Motivation . . . . . . . . . . . . . . . . . . . 20

4.2 Proposed Scheme: PNS Criteria . . . . . . . . . . . . . . . . 20

4.2.1 System Model . . . . . . . . . . . . . . . . . . . 20

4.2 .2 Data Sensing Phase . . . . . . . . . . . . . . 21

4.2.3 Depth Threshold Selection Phase . . . . . . . . . . . . . 21

4.2 .4 Data Routing Phase . . . . . . . . . . . . . . 21

4.2.4.1 PNS-DRE ..................... 21

4.2.4.2 PNS-DRE-SNR . . . . . . . . . . . . . . 22

4.3 Results and discussion . . . . . . . . . . . . . . . . . 24

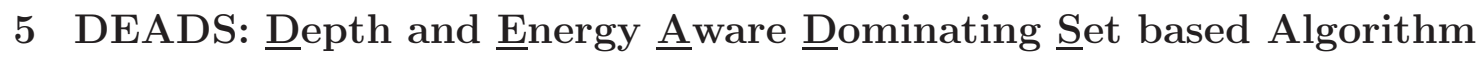
for Cooperative Routing along with Sink Mobility in Underwater WSNs 29 
5.1 Motivation . . . . . . . . . . . . . . . . . . . . 30

5.2 Channel Model . . . . . . . . . . . . . . . . . . . . . . . . . 30

5.3 Mathematical Model . . . . . . . . . . . . . . . . . . . . . . 31

5.4 DEADS: The Proposed Scheme . . . . . . . . . . . . . . . 38

5.4 .1 Data Sensing and Routing . . . . . . . . . . . 38

$5.4 .2 \quad$ Sink Mobility . . . . . . . . . . . . . . . . 40

5.4.2.1 Linear Mobility Pattern . . . . . . . . . . . . . 40

5.4.2.2 Elliptical Mobility Pattern . . . . . . . . . . . . . 41

5.5 Simulation Results and Discussions . . . . . . . . . . . . . . . . 43

5.5 .1 Performance Tradeoffs Made by DEADS . . . . . . . . . 52

$\begin{array}{llr}6 & \text { Conclusion } & 55\end{array}$

$\begin{array}{lll}7 & \text { References } & 57\end{array}$ 


\section{LIST OF FIGURES}

3.1 Network architecture . . . . . . . . . . . . . . . . . . . . 9

3.2 Data packet format . . . . . . . . . . . . . . . . 12

3.3 Depth threshold selection $\left(d_{t h 1}=40, d_{t h 2}=30, d_{t h 3}=20\right) \ldots \ldots$

3.4 Comparison of alive nodes in the network . . . . . . . . . . . . . 16

3.5 Comparison of average energy consumption of nodes in the network . . . 17

3.6 Comparison of no. of packets delivered at sink . . . . . . . . . . 17

3.7 Comparison of end-to-end delay . . . . . . . . . . . . . . . . . . . 18

3.8 Comparison of transmission loss in the network . . . . . . . . . . . 18

4.1 System Model . . . . . . . . . . . . . . . . . . . . . . . . . 20

4.2 Depth threshold (highest $d_{t h}$ ) and cooperative partner nodes selection . . 23

4.3 Depth threshold (medium $d_{t h}$ ) and cooperative partner nodes selection . 23

4.4 Depth threshold (lowest $d_{t h}$ ) and cooperative partner nodes selection . . 23

4.5 Comparison of alive nodes in the network with elliptical sink mobility pattern 25

4.6 Comparison of total energy consumption of network with elliptical sink mobility pattern . . . . . . . . . . . . . . . 26

4.7 Comparison of packets received at MSs with elliptical sink mobility pattern 27

4.8 Comparison of packet drop with elliptical sink mobility pattern . . . . . 27

4.9 Comparison of PAR with elliptical sink mobility pattern . . . . . . . 28

5.1 Network model . . . . . . . . . . . . . . . . . . . . . . . . 31

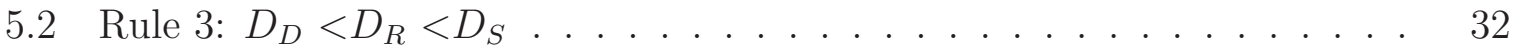

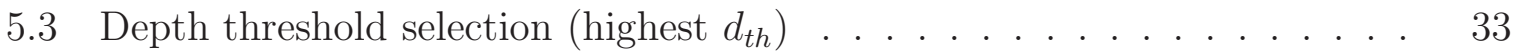

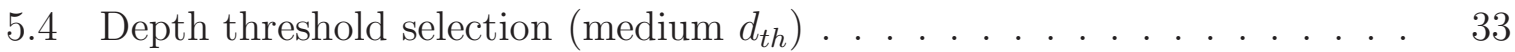

5.5 Depth threshold selection $\left(\right.$ lowest $\left.d_{t h}\right) \ldots \ldots$. . . . . . . . . . . . 34

5.6 Flowchart showing selection of relay and destination nodes in a particular sub-graph . . . . . . . . . . . . . . . . . . . 37

5.7 Selection of $D S$ and $C C$ nodes in a sub-graph . . . . . . . . . . . 38

5.8 Data routing in the network with linear sink mobility pattern . . . . . . 41

5.9 Specifications of elliptical mobility path followed by MSs . . . . . . . . . 42

5.10 Data routing in the network with elliptical sink mobility pattern . . . . . 43

5.11 Comparison of alive nodes in the network with linear sink mobility pattern 45 
5.12 Comparison of alive nodes in the network with elliptical sink mobility pattern 45

5.13 Comparison of total energy consumption of network with linear sink mo-

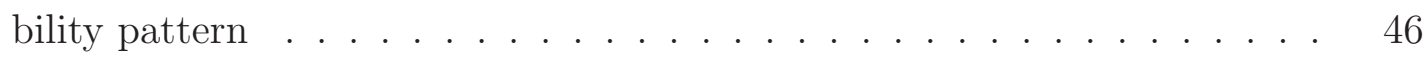

5.14 Comparison of total energy consumption of network with elliptical sink mobility pattern . . . . . . . . . . . . . . 47

5.15 Comparison of packets received at MSs with linear sink mobility pattern 48

5.16 Comparison of packets received at MSs with elliptical sink mobility pattern 48

5.17 Comparison of packet drop with linear sink mobility pattern . . . . . . 50

5.18 Comparison of packet drop with elliptical sink mobility pattern . . . . 50

5.19 Comparison of PAR with linear sink mobility pattern . . . . . . . . 51

5.20 Comparison of PAR with elliptical sink mobility pattern . . . . . . 52 


\section{LIST OF TABLES}

4.1 Simulation parameters ....................... 24

5.1 Analysis of existing depth-based routing protocols . . . . . . . . . . . 44

5.2 No. of alive nodes after equal time intervals (sec) . . . . . . . . . . . 46

5.3 Network's residual energy in joules after equal time intervals (sec) . . . . 48

5.4 Network's throughput in bits after equal time intervals (sec) . . . . . . . 49

5.5 No. of dropped bits after equal time intervals (sec) . . . . . . . . . . . 51

5.6 Performance comparison of DEADS-SRC and DEADS-MRC with counterpart techniques . . . . . . . . . . . . . . 53

5.7 Performance tradeoffs made by routing protocols . . . . . . . . . . . . 54 
Chapter 1

Introduction 
Oceans cover about two-third surface of the earth. It has gained importance because of the hidden resources, military surveillance purposes and underwater environment monitoring. UWSNs has gained attention for these explorations. Typical framework of UWSN is composed of underwater sensor nodes, sink nodes, surface station(s), etc. Acoustic signals are used for communication in underwater environment. The harsh underwater environment and unique characteristics of acoustic signals impose numerous challenges on efficient data routing in UWSNs. Sensor nodes consume more energy due to severe environmental conditions where replacement or recharging of battery is not possible. Bandwidth available for communication is limited i.e. $<100 \mathrm{kHz}$ because of attenuation and absorption of acoustic signals. Link characteristics like transmission loss, propagation delay and Signal to Noise Ratio (SNR) are continuously varying with time and depth. BER are high because of multipath fading and refraction phenomena. Therefore, high throughput, reduced delay, and energy efficiency along with robustness and reliability of retrieved data stand out as a critical consideration [1].

Graph theory serves to mathematically model different network structures. It is widely used to devise algorithms for efficient data routing in Wireless Sensor Networks (WSNs). UWSNs can be represented by a directed graph (digraph), $G=(V, A)$. A digraph is a graph, or set of nodes $(V)$ connected by directed edges $(A)$, where the directed edges have a direction associated with them. If $a=(u, v) \in A$, this means nodes $u$ and $v$ are able to directly communicate with each other. In formal terms, a digraph is a pair of:

- A set $(V)$, whose elements are called vertices or nodes.

- $\mathrm{A}$ set $(A)$ of ordered pair of vertices, called arcs, directed edges, or arrows.

We are considering a digraph for data routing in underwater environment because in depth-based routing data needs to be routed upwards, that is, towards low depth (station present on water surface).

Cooperative communication is incorporated in UWSNs to improve the network performance in terms of packet delivery ratio and data reliability. It exploits the broadcast nature of wireless transmission and relies on cooperation of nodes in relaying each others information. Cooperative transmission involves the use of a source, relay and destination node. When source node broadcasts any signal it is received by destination node and also overheard by relay node in its sensing range. Relay node then forwards this overheard information to destination node as a replica of the original signal [2]. Two types of relaying schemes mainly implemented at relay node are: Amplify-and-Forward (AF) and Decode-and-Forward (DF) [3]. Relay node simply amplifies the signal and transmits it in the former scheme, whereas, received signal is decoded, corrected, recoded and then transmitted in later. Different number of relay nodes can be selected as per requirement. 
Traditional model of UWSNs involves static sink (usually present on water surface), however, static sink results in hotspot problem. It is a problem in which sensor nodes closer to the sink tend to die early because of forwarding more packets(packets of nodes that lie far away from sink), leaving areas of network completely unmonitored. Hence, it is beneficial to use Mobile Sinks (MSs) to transfer sensed information [1].

In this work, we present a mathematical model for cooperative routing along with sink mobility in UWSNs. We propose an algorithm to compute the Dominating Set (DS) of a given network modelled as a digraph. DS is a subset of vertices $\mathrm{V}$ such that each node in V - DS is adjacent to at least one node in DS. DS formation helps in organizing nodes in a way that is optimal for cooperative routing. DS can be represented as a backbone which is a subset of nodes that are able to perform special tasks and can serve nodes that are not in the backbone. This backbone reduces communication overhead, increases bandwidth efficiency, decreases overall energy consumption, and, increases network lifetime in UWSNs. DS nodes while acting as a backbone efficiently forward data towards MSs which are following pre-defined optimal trajectories. Along with this, two different selection criteria, Partner Node Selection based on Depth Threshold and Residual Energy (PNS-DTRE) and Partner Node Selection based on Depth Threshold, Residual Energy and Signal to Noise Ratio (SNR) (PNS-DTRE-SNR) are also implemented and compared. PNS-DTRE considers a nodes residual energy and its depth threshold $\left(d_{t h}\right)$. Where, in our work $d_{t h}$ varies from node to node and depends on the number of a nodes alive neighbors. PNS-DTRE-SNR also takes SNR into consideration along with residual energy and $d_{t h}$. 
Chapter 2

Literature Review 
Cooperative communication has been proposed as a powerful alternative to reduce fading and other link impairments. It takes advantage of the broadcast nature of wireless transmission in which transmitted signal can be overheard by many unintended sensor nodes [2]. Some work done in this domain is presented here.

An innovative physical layer solution involving cooperative communication is given in [2], where outage probability and capacity expressions are derived for cooperative multicarrier Underwater Acoustic Communication (UAC) systems with AF and DF relaying. Further, they propose a receiver design to mitigate the degrading Doppler effects. In [3], Cooperative UnderWater Acoustic Multiple Input Single Output (CUWA-MISO) using $\mathrm{DF}$ is proposed where each node present in network uses nearest adjacent node as a virtual antenna in a cooperative manner. This process improves the system performance with the help of spatial diversity. Luo et al. [4], explore cooperation at Medium Access Control (MAC) layer and proposed a distributed MAC protocol, that is, Coordinated Transmission MAC (CT-MAC) for underwater MIMO based network uplink communication. The scheme also addresses long propagation delay and collision among control packets in UAC. Authors in [5] suggest that channel efficiency is improved by applying asynchronous cooperative transmission for three dimensional UWSNs. Two typical forwarding schemes: AF and DF are implemented, analyzed and compared. Relay Selection Scheme Based on Propagation Delay for Cooperative Underwater Acoustic Network is proposed in [6]. In this scheme, optimal number of relays is chosen on the basis of changing underwater circumstances. Propagation delay among the relay nodes is considered as a criterion for relay partner selection. SNR is also added to the criterion of relay selection to achieve further improvement in results. In COoperative Best Relay Assessment (COBRA) [7], a relay selection criterion for underwater cooperative acoustic networks is developed. Best relay selection algorithm is used here. This scheme requires statistical information of channel instead of instantaneous channel state. COBRA improves network performance in terms of throughput, delivery ratio and reduces large propagation delay. In [8], Cooperative routing along with sink mobility are implemented and analysed at the network layer. Nodes for cooperative routing are selected on the basis of their depth and residual energy information. In presence of mobile sink, data is directly forwarded towards it in a cooperative manner. Whereas, in absence of mobile sink, it is forwarded towards a nodes cooperative partners. In [9], CoDBR (Cooperative Depth Based Routing) is proposed that achieves information reliability and throughput efficiency. Potential relays for cooperative routing are selected on the basis of their respective depths. Data from source node is cooperatively forwarded towards destination via relay nodes. It achieves improvement in throughput and reduces packet drop as compared to the non-cooperative DBR.

Improving network lifetime is a fundamental challenge for UWSNs. One possible solution exists in making use of sink mobility. Some of the protocols presented in this domain are 
An adaptive strategy is proposed by authors in [1] that guarantees minimum energy consumption by enforcing sink redeployment. Sink redeployment decision is based on routing information. When there is high mobility, more nodes switch to use high power levels for communication. To overcome this, a sink redeployment procedure starts to find an optimal new location of surface sink. A coordination scheme using sink mobility for data gathering in UWSNs is proposed by authors in [10] that achieve energy efficiency and communication reliability. The shortest wakeup time and optimal period of sleep time for sensor nodes are investigated in this work. In [11], an underwater application model for collecting detailed data with multiple MSs to get high temporal resolution capability is presented. In [12], sink mobility is used to improve network lifetime in three dimensional UWSNs. Radius of monitored region grows in three dimensional networks, hence, improved lifetime and efficient energy utilization of nodes are of great concern. MobiRoute defined by authors in [13] supports sink mobility. Its favorable features are sinks controlled and predictable mobility and pause time. Autonomous Underwater Vehicle (AUV) aided Underwater Routing Protocol (AURP) [14] minimizes total number of data transmissions by using multiple MSs or AUVs as relay nodes, which results in a high packet delivery ratio and low energy consumption. In AUV aided Energy Efficient Routing Protocol (AEERP) for UWSNs [15], a MS collects data from gateway nodes. Among all the nodes, gateway nodes communicate with MS. Gateway nodes are selected on the basis of their residual energy.

Localization-free, non-cooperative depth-based routing protocols for UWSNs are: In [16], a novel routing protocol called Depth-Based Routing (DBR) is proposed that uses depth of sensor nodes as a routing metric and forwards data towards sink by applying greedy approach. In [17], an energy efficient routing protocol, named Energy-Efficient DepthBased Routing (EEDBR) is presented. EEDBR utilizes depth as well as residual energy of sensor nodes as routing metrics so that network lifetime can be improved. In Improved Adaptive Mobility of Courier Nodes in Threshold-Optimized DBR (iAMCTD) [18], a forwarding function based routing scheme is proposed. It is a novel network prototype in localization-free and flooding based routing for underwater applications. It achieves energy conservation. 


\section{Chapter 3}

On Enhancing Network Reliability and Throughput for Critical-Range Based Applications in UWSNs 


\subsection{Motivation and contribution}

iAMCTD adapts the depth threshold with varying network density. Hence, it requires the exchange of control information at regular intervals. This information exchange while acting as an overhead utilizes network resources. Although the stability period is much improved in iAMCTD, however, the trend of instability period is not balanced. It indicates a non-uniform reduction in network density per round. Moreover, the movement pattern of courier nodes is not optimized to reduce burden on medium depth nodes which greatly reduces throughput and overall network performance. In our work,

- The changes in depth threshold are independent of the network density information, avoiding the exchange of control packets throughout the network life time.

- The proficient movement of courier nodes minimizes the burden on medium depth nodes and decrements the energy consumption of low-depth nodes by utilizing onspot data collection which collectively increases throughput.

- Stability period is improved and the node density remains comparatively high indicating that the network is becoming sparse in a gradual manner which improves the overall network reliability.

Hence, we promote global load balancing in our proposed scheme by modifying the iAMCTD protocol for critical-range based applications. Based on the above analysis, this work presents a scheme on enhancing network reliability and throughput for critical-range based applications to accomplish resourceful energy expenditure of nodes in UWSNs.

\subsection{Proposed Scheme}

In this section we present our work in detail

\subsubsection{Network Architecture}

Figure 3.1 shows the network architecture of our proposed scheme. Numerous sensor nodes are randomly deployed in the area to be monitored. Sink nodes are deployed on the water surface and courier nodes are deployed in water at different depths. Courier nodes follow a horizontal pattern of movement. The sensor nodes use acoustic modems whereas the sink nodes and courier nodes are equipped with both acoustic and radio modems. As radio communication is 5 times faster than acoustic communication, a data packet once received at any sink or courier node is considered delivered to the on-shore data center. As depth of the courier nodes remains fixed, they relay data to the sink by 
forwarding it to other courier nodes having lesser depth. In the knowledge acquisition phase, nodes share their depth and residual energy information with each other.

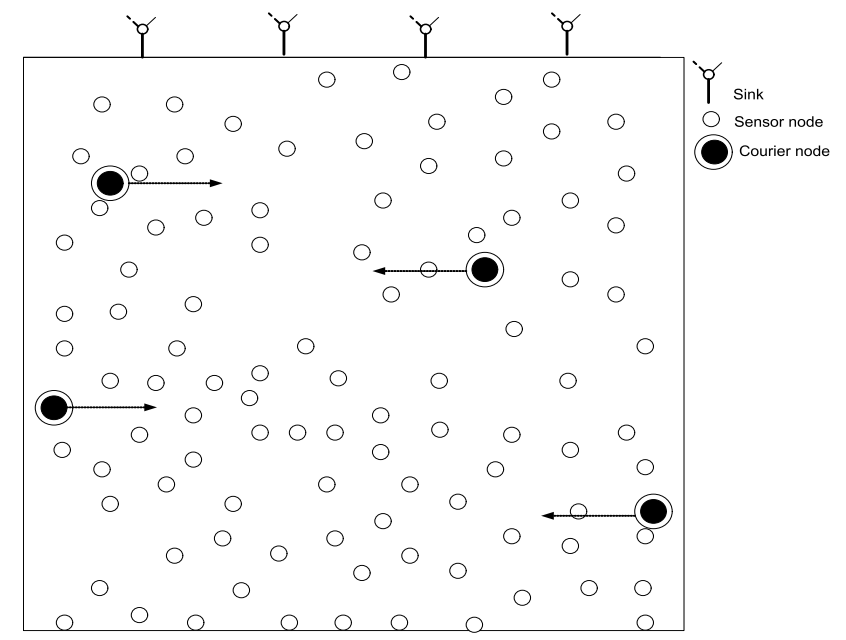

Figure 3.1: Network architecture

\subsubsection{Underwater Channel Model}

The channel model used to calculate signal attenuation and total noise loss in underwater acoustics is detailed here. We are interested in working out total attenuation of signal on the basis of spreading loss [19] and signal absorption loss. At a given frequency f, Thorps channel model calculates absorption loss $\alpha(\mathrm{f})[20]$ as follows:

$$
10 \log \alpha(f)=\left\{\begin{array}{cc}
0.11 f^{2} /\left(1+f^{2}\right)+44 f^{2} /(4100+f) & \\
+2.75 * 104 f^{2}+0.003 & f \leq 0.4 \\
0.002+0.11(f /(1+f))+0.011 f & f<0.4
\end{array}\right.
$$

Here $\alpha(\mathrm{f})$ is in $\mathrm{dB} / \mathrm{km}$ and $\mathrm{f}$ in $\mathrm{kHz}$. We determine the value of $\alpha$ by using the value of absorption loss, as follows:

$$
\alpha=10^{\alpha(f)} / 10
$$

likewise, all tunable parameters are given in $\mathrm{dBre} \mu \mathrm{Pa}$. The total attenuation $\mathrm{A}(\mathrm{l}, \mathrm{f})$ can be computed by combining absorption loss and spreading loss, as follows:

$$
10 \log (A(l, f))=k * 10 \log (l)+l * 10 \log (\alpha(f))
$$

Where, first term corresponds to spreading loss and the second term to the absorption loss. The spreading coefficient $\mathrm{k}$ defines the geometry of the signal propagation in underwater acoustics (i.e., $\mathrm{k}=1$ for cylindrical, $\mathrm{k}=2$ for spherical, and $\mathrm{k}=1.5$ for practical spreading $[21])$. 
The ambient noise in underwater networks is composed of turbulence noise, shipping noise, wind noise and thermal noise. The power spectral density [19] of the four noise components in $\mathrm{dBre} \mu \mathrm{Pa}$ is given by the following formulae as a function of frequency in $\mathrm{kHz}$ :

$$
\begin{gathered}
10 \log \left(N_{t}(f)\right)=17-30 \log (f) \\
10 \log \left(N_{s}(f)\right)=40+20(s-0.5)+26 \log (f)-60 \log (f+0.03) \\
10 \log \left(N_{w}(f)\right)=50+7.5 w^{1 / 2}+20 \log (f)-40 \log (f+0.4) \\
10 \log \left(N_{t h}(f)\right)=-15+20 \log (f)
\end{gathered}
$$

where $N_{t}, N_{s}, N_{w}$ and $N_{t h}$ represent turbulence noise, shipping noise, wind noise and thermal noise. The overall noise power spectral density [22] can be calculated as:

$$
N(f)=N_{t}(f)+N_{s}(f)+N_{w}(f)+N_{t h}(f)
$$

\subsubsection{Network Initialization Phase}

Because of the knowledge acquisition phase, all nodes are aware of each others' depth. When the network starts, nodes find their neighbors. Any node selects that node as its neighbor that is within its transmission range and is having comparatively lesser or equal depth. Based on the number of neighbors of a node $d_{t h}$ is selected. $d_{t h}$ is used to avoid flooding. After the $d_{t h}$ is selected, nodes perform data forwarding on its basis. In this way, global knowledge of network density is not required and nodes adapt their $d_{t h}$ based on their alive neighbors.During this phase, each node is advised to keep on identifying its alive neighbors at regular intervals to adapt its $d_{t h}$ accordingly.

\subsubsection{Critical-range Based Data Sensing}

Traditional schemes consumed surplus energy because of transmission of redundant data and retransmission of sensitive data. We exploited critical-range based data sensing and routing. This critical-range is specified on an application basis.Algorithm 1 depicts the involved procedure. $\mathrm{S}$ is the sensed attribute, $S_{t h}$ and $H_{t h}$ specify lower and upper limits of the critical range. $R_{i}$ represents the residual energy of the $i_{t h}$ node. After network initialization, nodes start sensing the specified environmental attribute. If the sensed value is greater than or equal to $H_{t h}$, flag field of the data packet is set equal to 1 . Then, this data packet is forwarded if node's residual energy $R_{i}$ is sufficient for transmission. The other scenario is that if the sensed value is lesser than $H_{t h}$ but greater than or equal to $S_{t h}$. In this scenario, node sets flag field of the data packet equal to 0 and performs forwarding if it is having enough residual energy $R_{i}$. Here, flag field is appended to 
the basic packet structure in order to indicate severity of the sensed attribute, so that appropriate measures can be adopted at the on-shore data center.

The proposed scheme is applicable for many scientific applications that observe the environment; from geological processes on the ocean floor, to water characteristics (temperature, salinity, oxygen level, bacterial and other pollutant content, dissolved matter, etc.) to counting or imaging animal life (microorganisms, fish or mammals) [23].

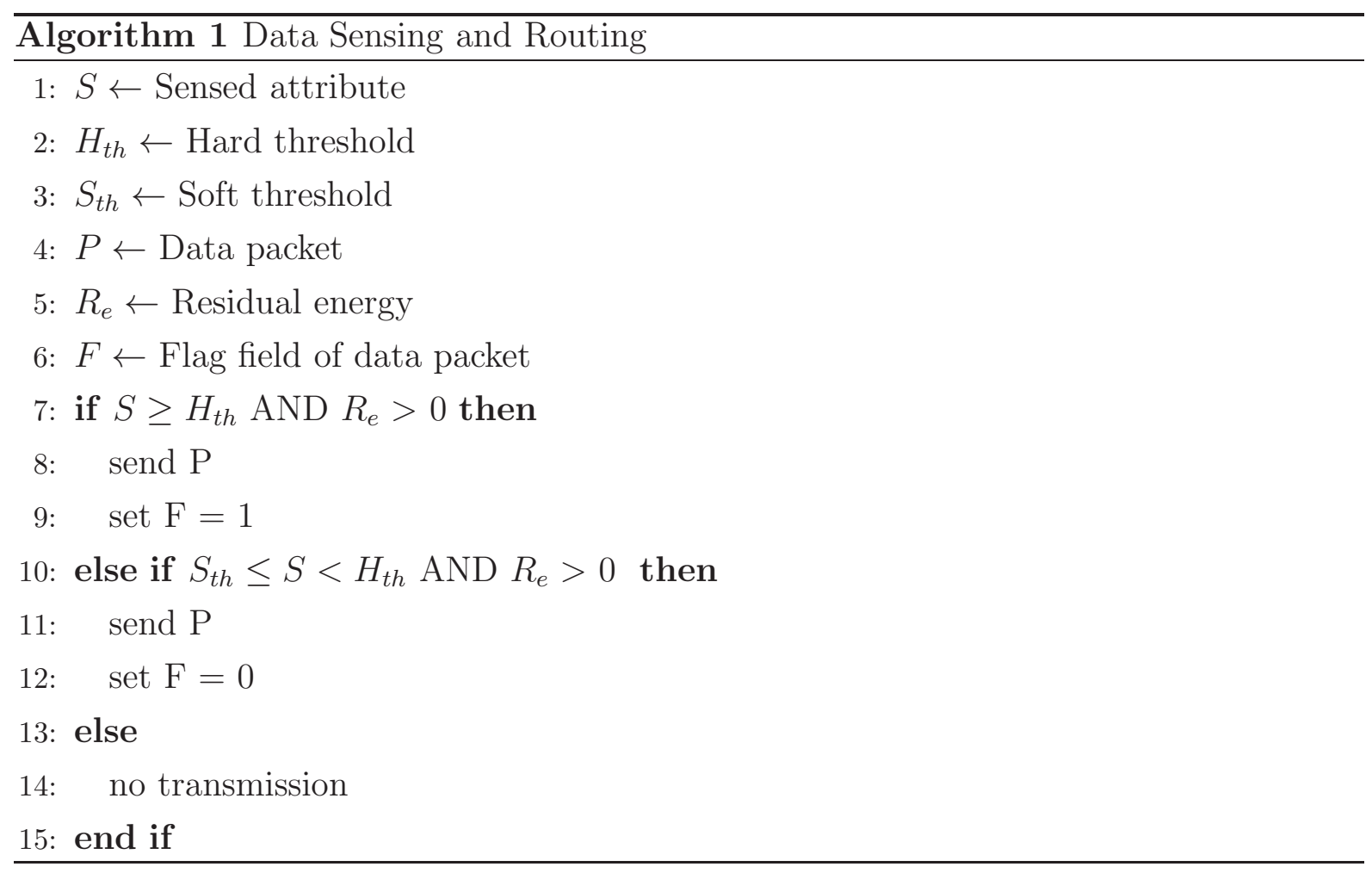

\subsubsection{Data Routing Phase}

Data generated as a result of the sensing process is to be reported to the sink. For this purpose, a source node forwards data packets to in-range courier node. On reception of data, courier node commands the source node to avoid further flooding of this data. In absence of courier node, source node forwards data to its depth threshold based neighbors. Receiving(relaying) nodes keeps the received packets in buffer and performs forwarding on the basis of holding time. Holding time is calculated by using the forwarding function. Forwarding function is depth dependent and is different for nodes with different depths. Also, priority queue is maintained at relaying nodes in order to avoid transmission of same data packets. This mechanism reduces end-to-end delay of critical data.

Data packet is composed of header and payload. Header contains the control information necessary for data routing whereas payload is the actual data to be transmitted. Data packet format used in our scheme is shown in figure 3.2 


\begin{tabular}{|l|l|l|l|l|}
\hline Flag & Sender ID & Packet Sequence Na & Depth & Payload \\
\hline
\end{tabular}

Figure 3.2: Data packet format

\subsubsection{Depth Threshold Selection Process}

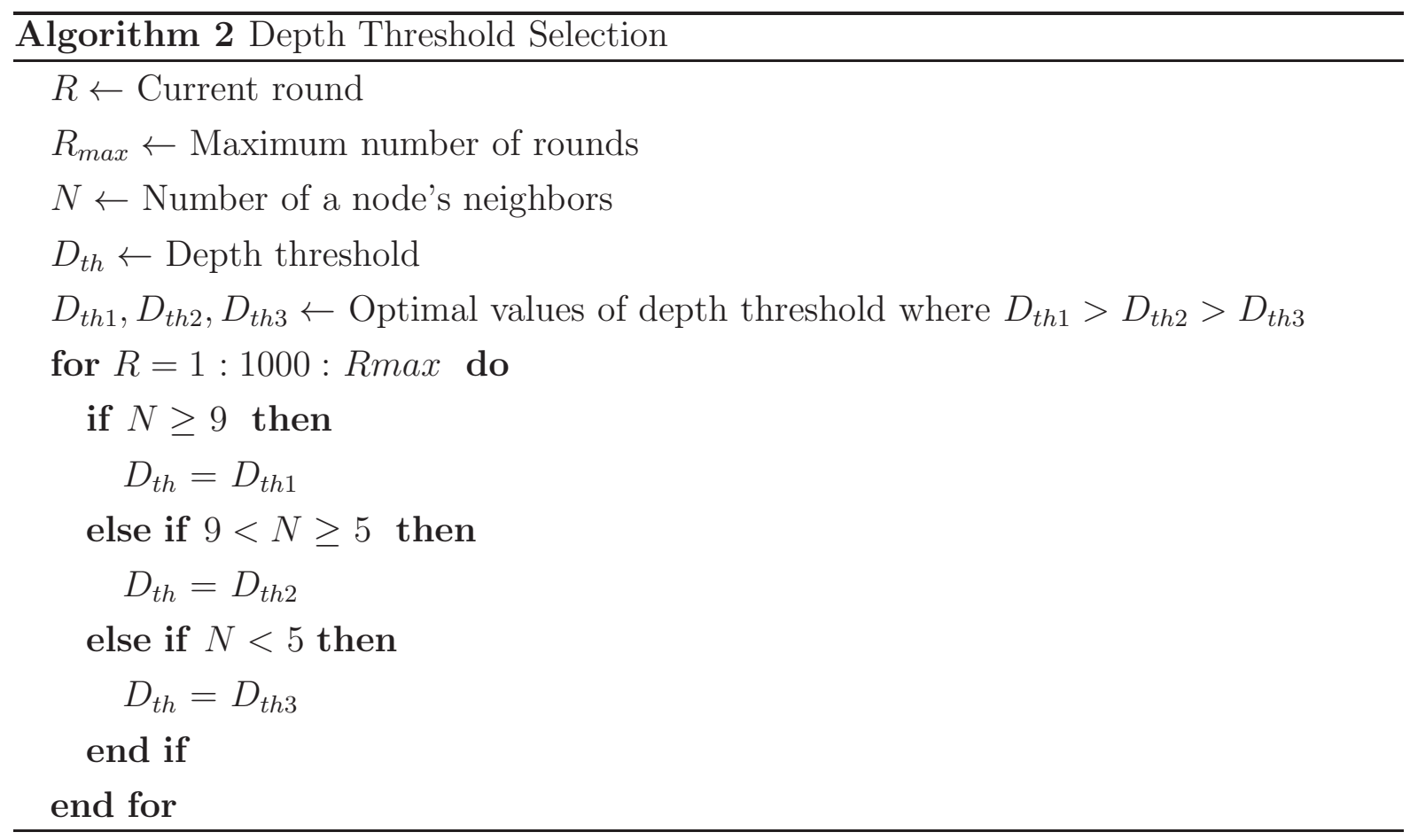

During the network initialization phase, each node is advised to keep on identifying its alive neighbors at regular intervals to adapt the depth threshold accordingly. Depth threshold selection process of nodes is shown in figure 3.3. This procedure is detailed in algorithm 2. Lifetime of the network is divided into rounds. The three optimal values of depth threshold $\left(D_{t h 1}, D_{t h 2}\right.$ and $\left.D_{t h 3}\right)$ are adapted on the basis of number of alive neighbors of a node. The whole procedure is shown in algorithm 2 . 


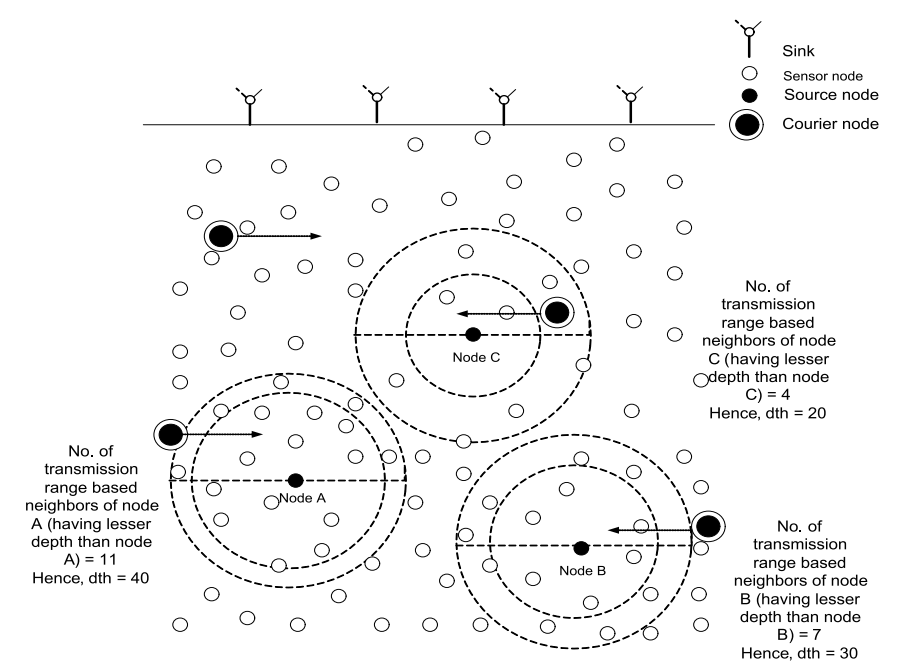

Figure 3.3: Depth threshold selection $\left(d_{t h 1}=40, d_{t h 2}=30, d_{t h 3}=20\right)$

\subsubsection{Mobility Pattern of Courier Nodes}

Introduction of courier nodes in UWSNs is an ingenious way of improving network stability and reliability. Deployment of courier nodes in a network avoids distant transmissions and greatly reduces load on medium-depth nodes by acting as a relay. Sojourn tour specified by the sink defines the mobility pattern of a courier node. In our scheme, a horizontally varying mobility pattern is used that remains fixed throughout the network lifetime. After network initialization, courier nodes begin their movement and collect data from nodes in their vicinity. Mechanical modules are used by courier nodes to maintain their depth and movement pattern [24].

\subsubsection{Holding Time Computation on the Basis of Forwarding Function}

Holding time is the time for which the node holds the data packets in its buffer before forwarding it. It is given by the following formula:

$$
H_{T}=\left(1-F_{F}\right) H_{t \max }
$$

where $H_{T}$ represents the holding time and $F_{F}$ represents the value of forwarding function. $H_{\text {tmax }}$ denotes the maximum holding time and it is selected according to the environmental conditions.

At the relaying nodes, holding time is computed to transmit the received packets by utilizing forwarding function $F_{F}$. A node selects its $F_{F}$ on the basis of its depth, residual energy and SNR ratio. There are three main $F_{F}$ : Signal Quality Index (SQI), Energy Cost Function (ECF) and Depth Dependent Function (DDF) [18]. Among the set of 
neighboring nodes, node having highest $F_{F}$ acts as an optimal forwarder because of having minimum holding time. Prevailing conditions of the aqueous environment are considered while formulating the forwarding functions.

Upper region of water suffers from maximum interference, noise and shipping activities. Hence, to reduce the effect of these losses on signal strength, SQI is used as a forwarding function. It is represented as

$$
S Q I=\left(\operatorname{LSNR}\left(R_{i}\right)\right) / l_{i}
$$

where LSNR is the localization free signal to noise ratio, $R_{i}$ denotes the residual energy of $i_{t h}$ receiver node and $l_{i}$ is the depth difference between sender and receiver node.

There is a huge burden of data forwarding on nodes lying in medium depth region. Hence, to utilize their energy efficiently while taking into account their residual energy, ECF is used as the forwarding function to attain global load balancing. ECF is represented as

$$
E C F=\text { priority }_{\text {value }}\left(R_{i}\right) / D_{i}
$$

where priority $_{\text {value }}$ is a constant that can be adjusted according to stability requirements, $D_{i}$ is the depth of the $i_{t h}$ receiver node and $R_{i}$ is its residual energy.

Nodes lying in high depth region have to perform distant transmissions. Side by side, flooding is more prominent in this region. Hence, to reduce these phenomenons, DDF acts as the forwarding function which takes into account both signal strength and residual energy while selecting the optimal forwarder. Following is the expression for DDF

$$
D D F=L S N R\left(R_{i}\right) / D_{i}
$$

where LSNR is the localization free signal to noise ratio, $D_{i}$ is the depth of the $i_{t h}$ receiver node and $R_{i}$ is its residual energy.

A simple algorithm for data forwarding using $F_{F}$ is given in algorithm 3 


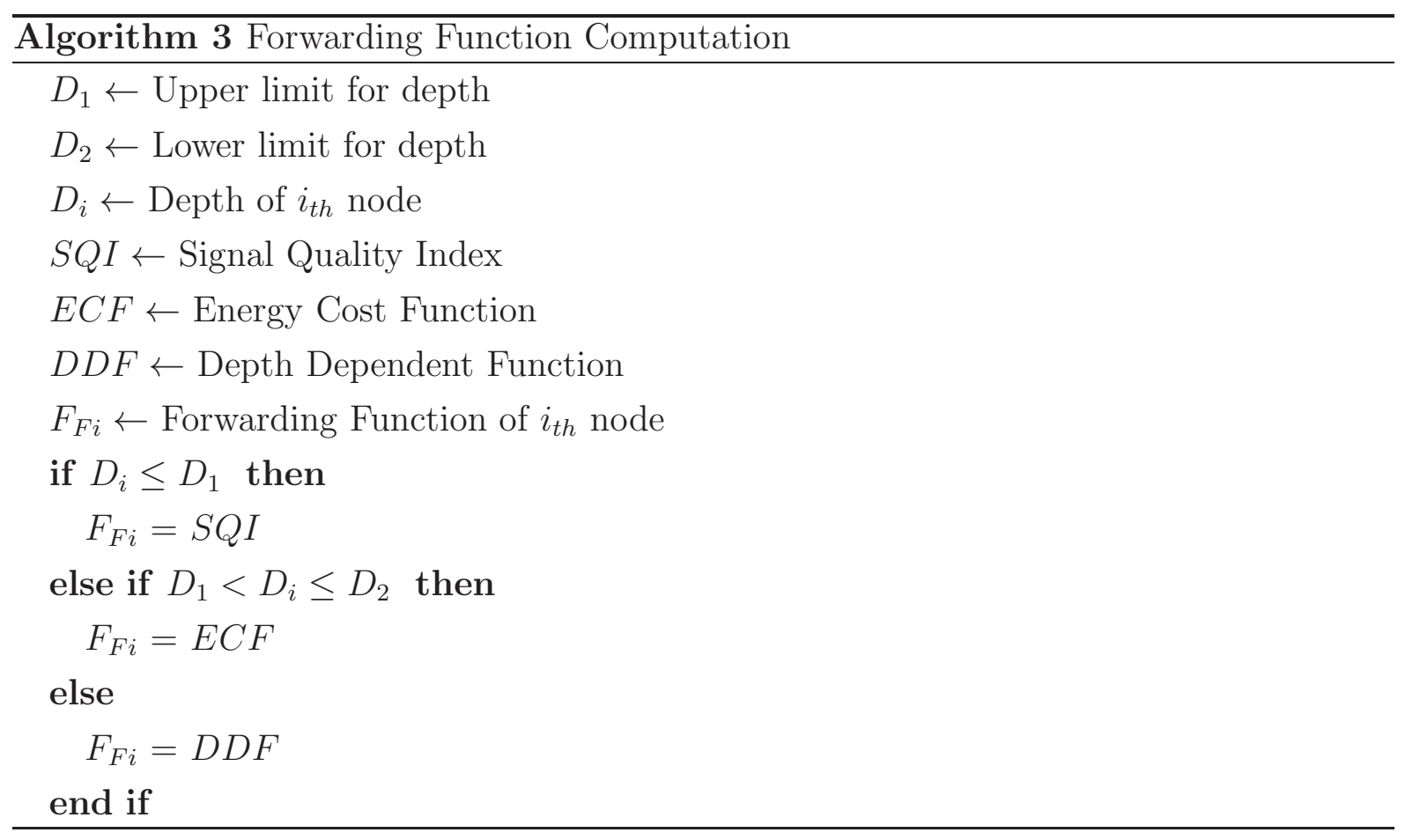

\subsection{Simulation Results}

We performed our simulations in MATLAB and compared our proposed scheme with iAMCTD. Sensor nodes are 225 in number and they are randomly deployed in underwater environment at an area of 500m x 500m. Four sinks are located on the water surface and they are $100 \mathrm{~m}$ apart from each other. Four courier nodes are deployed in water at different depths and they are following a horizontal pattern of movement. Each node is equipped with an initial energy of 5 Joules and is having a fixed transmission range of $100 \mathrm{~m}$. Size of data packet is 50 byte. The acoustic modem used is LinkQuest UWM1000 [25] which is having a bit rate of $10 \mathrm{kbps}$. Power consumption of a node in transmitting, receiving and idle modes is $2 \mathrm{~W}, 0.1 \mathrm{~W}$ and $10 \mathrm{~mW}$ respectively. Three different depth thresholds are used. During the network initialization phase, nodes select their depth threshold. Network lifetime is represented in terms of rounds where a round is a smallest cycle during which complete network operation is performed. After every span of 1000 rounds, each node recalculates its alive neighbors and changes its depth threshold accordingly.Following evaluation metrics are considered:

- stability period: It is a span in terms of rounds for which all nodes in the network remain alive. Stability period increases if a balanced energy consumption is involved in the network. A longer stability period avoids formation of coverage holes in the network.

- Alive nodes: It shows the number of nodes who are having sufficient residual energy. 
It basically represents network lifetime. Network lifetime increases when a balanced energy consumption is involved in the network. Presence of mobile entities for data collection also improves network lifetime by directly collecting data from nodes. Along with this, energy is conserved by avoiding flooding in the network which in turn increases network lifetime.

- Average energy consumption: It is the average energy consumed by all alive nodes per round. Energy is conserved by avoiding flooding and by introducing mobile entities for data collection in the network.

- Throughput: It is the total number of packets received at sink per round. Throughput increases selection of optimal forwarder node having best link state at any time instant. Short distance transmissions also increase throughput. Shorter is the distance between two nodes, lesser are the chances of data being corrupted by link impairments and higher is the throughput. Implementation of MRC as a diversity combining technique at next hop node also increases throughput.

- End-to-end delay: It represents the average time taken by packet to travel from source to sink. It is the ratio of distance between nodes and speed of acoustic signal. More is the distance between nodes more is the delay involved in data reception.

- Transmission loss: It shows the average transmission loss(dBs) between source node and sink in one round. Transmission loss depends on frequency of operation and distance between source node and sink. It increases if data has to traverse longer distances.

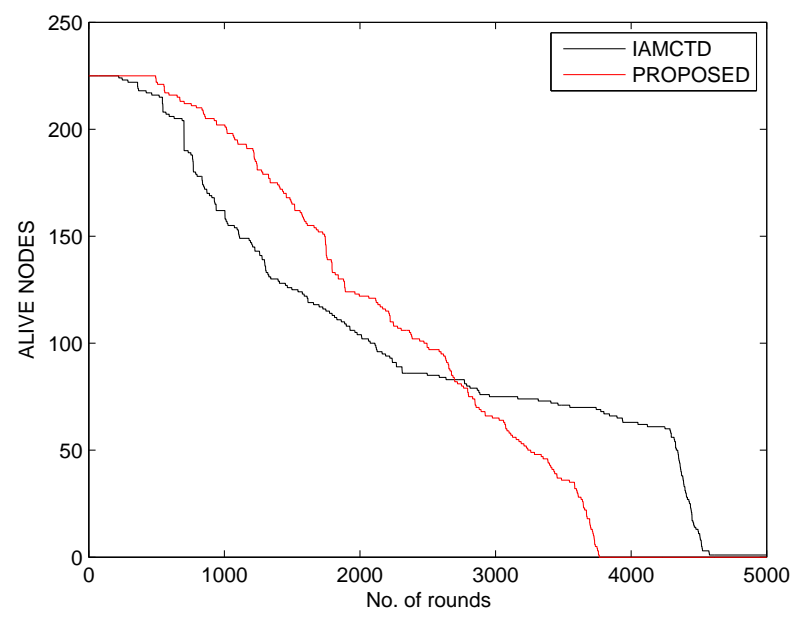

Figure 3.4: Comparison of alive nodes in the network

Highest energy consumption is involved in transmission. Throughput is high in our scenario indicating more number of transmissions. Because of this the network lifetime is 


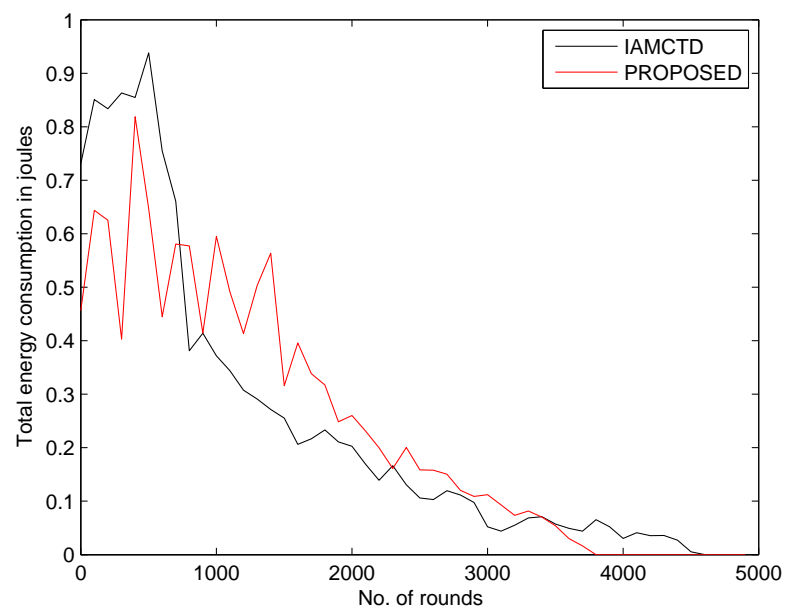

Figure 3.5: Comparison of average energy consumption of nodes in the network

reduced as compared to iAMCTD. However, as shown in figure 3.4, the stability period and trend of alive nodes per round is better in our scheme. The node density per round is comparatively high up till 2800 rounds indicating that the network is becoming sparse in a gradual manner improving the overall network reliability.

Figure 3.5 shows the comparison of average energy consumption of nodes in the network. Energy consumption is high in our scheme because of more number of transmissions.

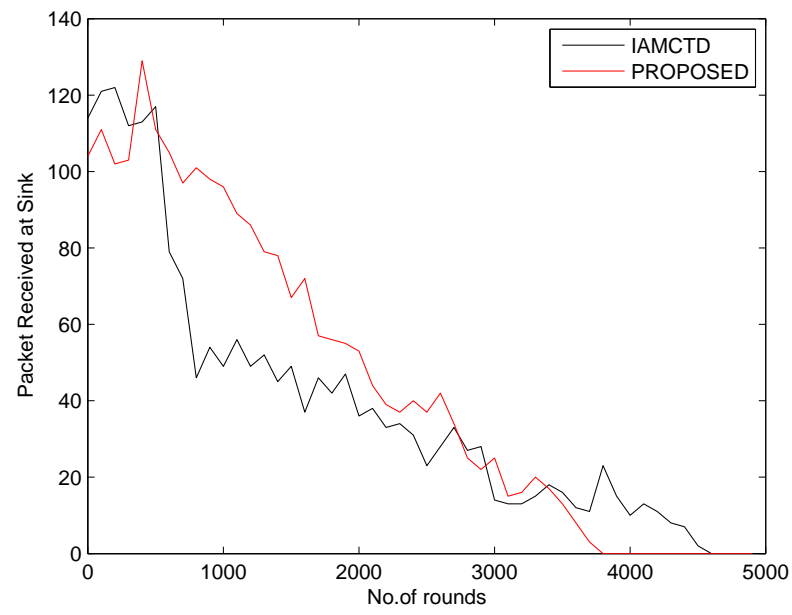

Figure 3.6: Comparison of no. of packets delivered at sink

Throughput is the number of packets received at sink. As shown in figure 3.6, throughput is increased in our scheme because of selection of optimal data forwarders (by varying the depth threshold according to a nodes alive neighbors) and favourable mobility pattern of courier nodes.

End-to-end delay refers to the time taken by a packet to be transmitted across the network from source node to sink. It is basically the ratio of time taken by the packet to reach 


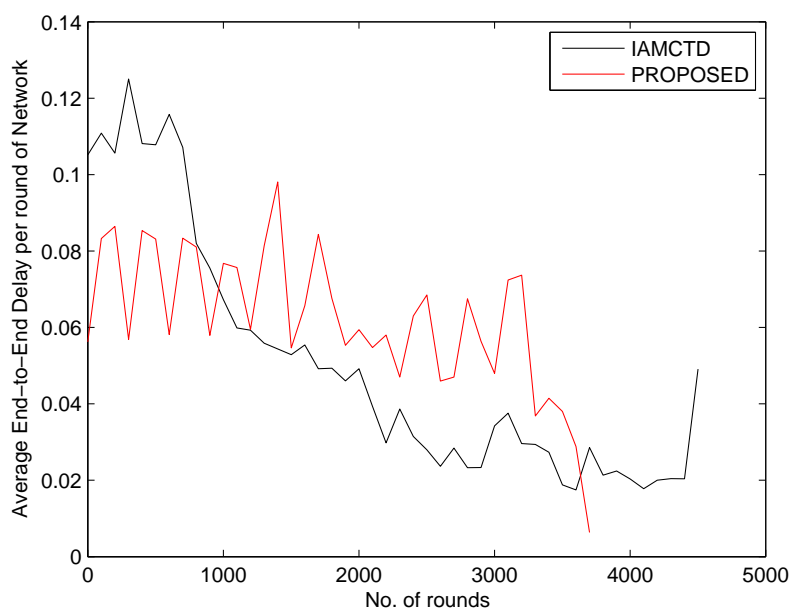

Figure 3.7: Comparison of end-to-end delay

to its destination and the number of packets transmitted per round.As shown in figure 3.7, it follows almost the same trend throughout the network lifetime in our scheme. As the throughput is high in our scenario, the end-to-end delay is comparatively low for up to 1100 rounds and follows almost the same pattern afterwards. In iAMCTD throughput decreases drastically after about 600 rounds because of which the end-to-end delay is reduced, whereas in our scheme it remains stable because of maintaining a high throughput.

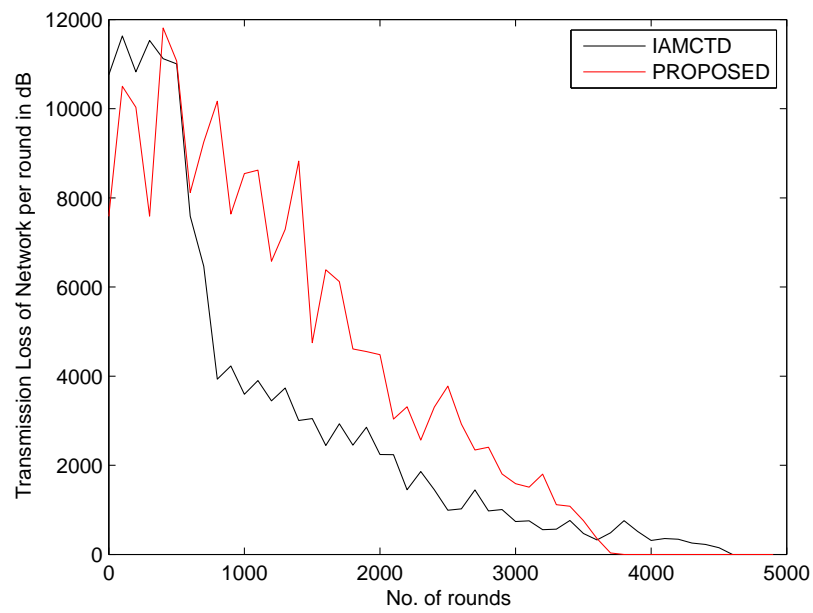

Figure 3.8: Comparison of transmission loss in the network

Transmission loss is distance dependent. Figure 3.8 shows that high throughput is achieved in compromise to higher transmission loss, as the number of transmissions between source nodes and sink are increased in our scheme. 


\section{Chapter 4}

\section{Partner Node Selection Criteria for Cooperative Routing in UWSN}




\subsection{Motivation}

Cooperative routing requires certain criterion on basis of which partner node are to be selected. As a selection criterion, only depth and residual energy of nodes are not reliable parameters. While considering depth and residual energy as routing metrics, link state is not taken into account. There is need of a certain criterion which also takes link quality into consideration. Link state information in terms of SNR states reliability and quality of the link. Hence, more appropriate partner node can be selected by considering a node's link state information along with its depth and residual energy as selection parameters. If link state between transmitter and receiver is good and SNR is high, then the chances of successful data transmission are much improved. Therefore, in this work, we focus on implementing and comparing these selection criteria for partner node.

\subsection{Proposed Scheme: PNS Criteria}

In this section, we present the two PNS criteria in detail.

\subsubsection{System Model}

System model comprises of randomly deployed sensor nodes that act as either source, destination and relay node. Fig. 4.1 shows the proposed system model for a single source destination pair. The links interconnecting these nodes suffer Rayleigh fading and Additive White Gaussian Noise (AWGN) noise. Binary Phase Shift Keying (BPSK) modulation is used in our scheme. A general mathematical model is represented by equation 4.1.

$$
Y_{n}=X \cdot g_{n}+N_{n}
$$

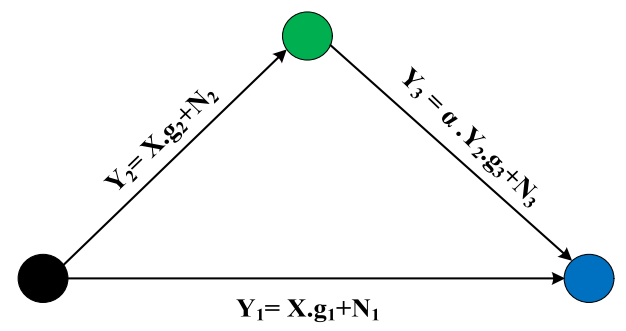

Figure 4.1: System Model

where $\mathrm{n}=1-3 . \mathrm{Y}$ is the signal received at relay and destination nodes. $\mathrm{X}$ is the original signal which is transmitted by source node, $\mathrm{g}$ is link gain and $\mathrm{N}$ is channel noise. AF is 
used as a relaying at relay node. Maximal Ratio Combining (MRC) is used as a diversity combining technique at the destination node. This scheme is based on the assumption that all the nodes are in perfect synchronization with each other.

System also involves multiple Mobile Sinks (MSs) intended to collect data from nodes. These MSs follow pre-defined trajectories and collect data from nodes in their vicinity.

\subsubsection{Data Sensing Phase}

Threshold optimized data sensing is used in our scheme as used in [8]. As the network starts, nodes start the sensing process. Whenever conditions specified by pre-defined hard and soft thresholds are met data packets are generated.

\subsubsection{Depth Threshold Selection Phase}

Depth threshold $\left(d_{t h}\right)$ is a parameter used to avoid flooding. It basically restricts the number of nodes involved in data forwarding [16]. In our scheme, we changed $d_{t h}$ on basis of a node's alive neighbors (nodes present within transmission range). Higher is the number of nodes, higher is the value of $d_{t h}$ and vice versa. When a node becomes aware of its $d_{t h}$, it divides its neighbor set into two. One comprises of nodes that lie within the boundary specified by $d_{t h}$ and the other set includes nodes that lie outside the $d_{t h}$ boundary but within its transmission range.

Nodes perform recalculation of $d_{t h}$ at regular intervals in order to adapt its value according to the number of alive neighbors.

\subsubsection{Data Routing Phase}

Sensed data is to be routed toward MSs so that it can be reported to surface station and finally to the on-shore data center. If a source node finds MS within its transmission range it forwards its data towards the MS in a cooperative manner. Whereas, in absence of MS, data is forwarded towards the selected destination node in a cooperative manner. This subsection describes the procedure involved in the selection of relay and destination node. Two criteria are explained below.

\subsubsection{PNS-DRE}

In this criterion, depth and residual energy are considered for partner node selection. Relay node is the one that lies within the boundary specified by $d_{t h}$ (lying at compara- 
tively lower, equal or higher depth)and possesses the highest residual energy. Destination node is the one that lies outside the boundary specified by $d_{t h}$ (lying at comparatively lower depth) and possesses the highest residual energy. After selection of partner node, cooperative routing is performed.

\subsubsection{PNS-DRE-SNR}

In this criterion, link state in terms of SNR is also incorporated along with residual energy and depth for partner node selection. In depth-based protocols, nodes are aware of their depth instead of location. Hence, Localization-free Signal-to-Noise Ratio (LSNR) represented by eq. 4.2 is used here [18].

$$
L S N R=P_{t} /((A(l, f) N(f))
$$

where $P_{t}$ is the constant transmitted power, attenuation noise (AN factor) is the product of path loss and ambient noise existing over any particular link and $\mathrm{f}$ is frequency of signal in $\mathrm{kHz}$.

In this criterion, network is divided into three distinct regions on basis of depth, that is, low, medium and high depth regions. Different LSNR-based functions are defined for these regions [18]. Signal Quality Index (SQI), Energy Cost Function (ECF) and Depth Dependent Function (DDF) represented by eq. 4.3, eq. 4.4 and eq. 4.5 are defined for low, medium and high depth regions. Nodes lying in respective regions are assigned weights on basis of these functions.

$$
\begin{gathered}
S Q I=\left(\operatorname{LSNR}\left(R_{i}\right)\right) / l_{i} \\
E C F=\text { priority }_{\text {value }}\left(R_{i}\right) / D_{i} \\
D D F=L S N R\left(R_{i}\right) / D_{i}
\end{gathered}
$$

where $R_{i}$ denotes residual energy of any $i_{t h}$ receiver node, $l_{i}$ is the depth difference between sender (source) receiver (relay, destination) nodes, $D_{i}$ is the depth of the sender

(source) node and priority $_{\text {value }}$ is a constant which can be adjusted according to stability requirement.

In this criterion, relay node is the one that lies within the boundary specified by $d_{t h}$ and possesses the highest weight. Similarly, destination node is the one that lies outside the boundary specified by $d_{t h}$ and possesses the highest weight. After selection of partner 
node, cooperative routing is performed.

Different cooperative routing scenarios for the above criteria are shown in fig. 4.2, 4.3 and 4.4. $d_{t h}$ varies with varying number of neighbors and relay/destination node is selected on the basis of residual energy/weight.

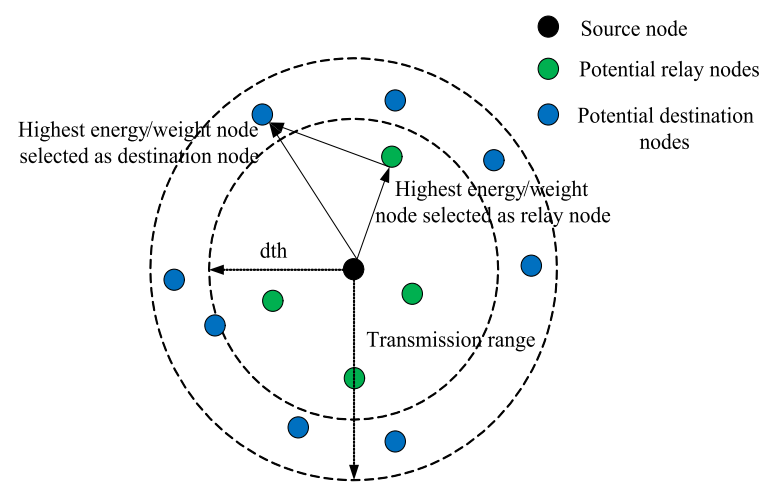

Figure 4.2: Depth threshold (highest $d_{t h}$ ) and cooperative partner nodes selection

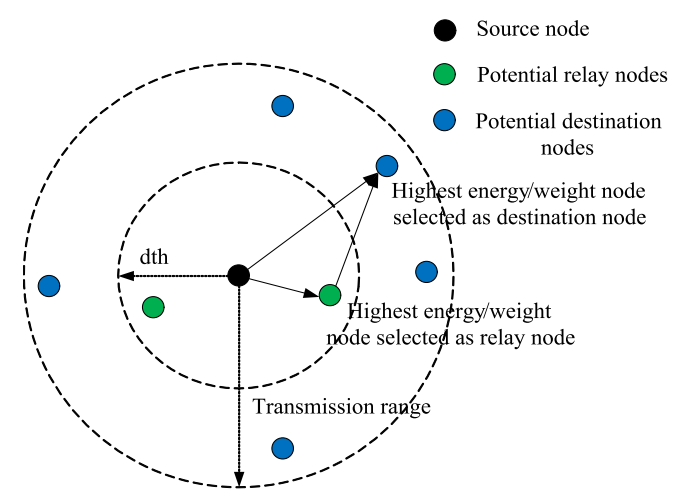

Figure 4.3: Depth threshold (medium $d_{t h}$ ) and cooperative partner nodes selection

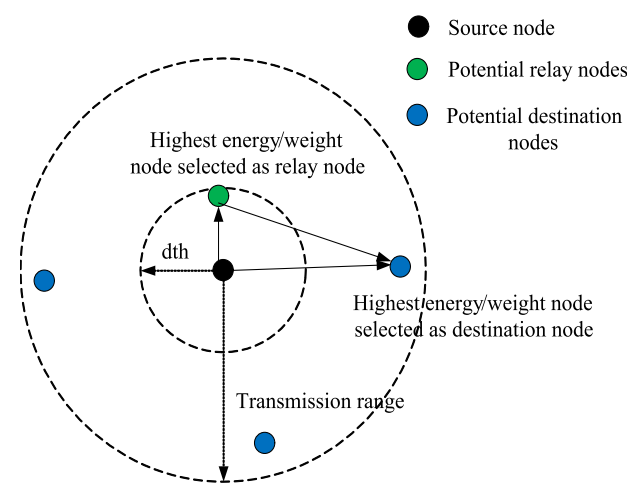

Figure 4.4: Depth threshold (lowest $d_{t h}$ ) and cooperative partner nodes selection 


\subsection{Results and discussion}

We perform simulations of our proposed scheme and compare it with already existing depth-based routing protocols, i.e., DBR and EEDBR. Different simulation parameters are summarized in table 4.1. The acoustic modem used is LinkQuest UWM1000 [25] which has a bit rate of $10 \mathrm{kbps}$. Power consumption of a node in transmitting, receiving and idle mode is $2 \mathrm{~W}, 0.1 \mathrm{~W}$ and $10 \mathrm{~mW}$ respectively.

Table 4.1: Simulation parameters

\begin{tabular}{|c|c|}
\hline Parameters & Value \\
\hline Number of Nodes & 225 \\
\hline Number of Sinks & 4 \\
\hline Area & $500 \mathrm{~m} \times 500 \mathrm{~m}$ \\
\hline Transmission Range & $100 \mathrm{~m}$ \\
\hline Initial Energy & 10 Joules \\
\hline Data packet & 1600 bits \\
\hline Control Packet & 48 bits \\
\hline
\end{tabular}

DBR considers depth and EEDBR considers depth along with residual energy. In our work, depth, residual energy and LSNR are the ultimate forwarding node determining factors. Following evaluation metrics are considered:

- Stability period: It is a span in terms of rounds for which all nodes in the network remain alive. Stability period increases if a balanced energy consumption is involved in the network. A longer stability period avoids formation of coverage holes in the network.

- Alive nodes: It shows the number of nodes who have residual energy that is sufficient for data transmission. It basically represents network lifetime. Network lifetime increases when a balanced energy consumption is involved in the network. Presence of mobile entities for data collection also improves network lifetime by directly collecting data from nodes. Along with this, energy is conserved by avoiding flooding in the network which in turn increases network lifetime.

- Residual energy: It is the residual energy possessed by all alive nodes per round. Energy is conserved by avoiding flooding and by introducing mobile entities for data collection in the network.

- Throughput: It is the total number of packets received at sink per round. Throughput increases selection of optimal forwarder node having best link state at any time instant. Short distance transmissions also increase throughput. Shorter is the distance between two nodes, lesser are the chances of data being corrupted by link 
impairments and higher is the throughput. Implementation of MRC as a diversity combining technique at next hop node also increases throughput.

- Packet drop: It shows the number of dropped packets out of total number of packets generated per round. If link state is not taken into account while selecting forwarder nodes then chances of packet drop are high. Long distance transmission also increases packet drop.

- PAR: It is defined as the ratio of total no. of packets received at the sink to the total no. of packets sent to the sink per round. Higher is the throughput more is the PAR and vice versa.

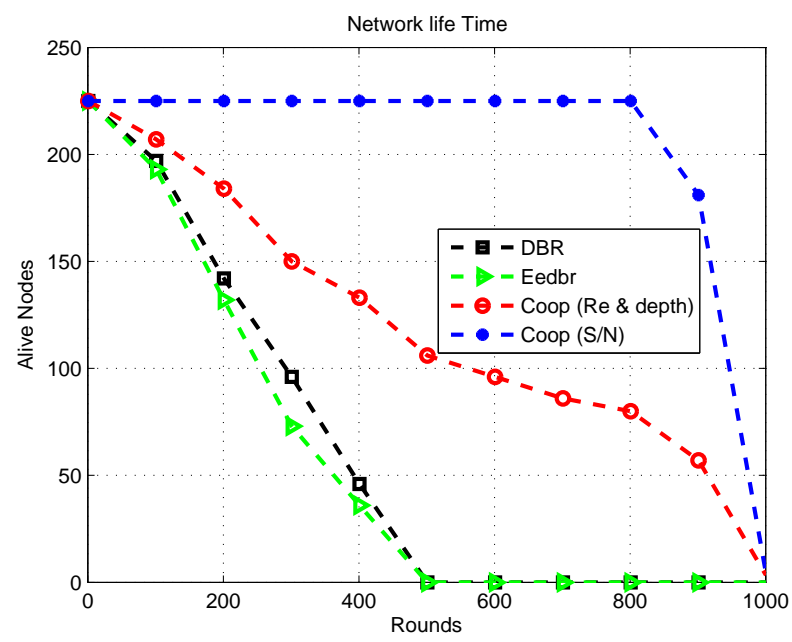

Figure 4.5: Comparison of alive nodes in the network with elliptical sink mobility pattern

As shown in fig. 4.5, in DBR and EEDBR, there is a small stability period, however, the instability period is much better in DBR as compared to EEDBR as there is gradual increase in energy consumption in DBR. When the network becomes sparse, number of neighbors decreases quickly causing network instability. In DBR, low-depth nodes die at an earlier stage due to high data forwarding rate and constant depth threshold. It neglects residual energy of nodes as well as link state thus badly affecting networks throughput. Number of dead nodes sharply increases in EEDBR as there is high load on high energy nodes. In residual energy and $d_{t h}$ based cooperative criterion, nodes begin to die very soon. This is because only residual energy is not a reliable parameter and does not provide any information regarding link state. SNR based criterion gives an ideal response in terms of large stability period. The reason is that it makes use of link state, depth, and depth differences as cooperative partner node selection parameters which in turn prevents the creation of coverage holes and nodes density remains constant throughout the network lifetime. 


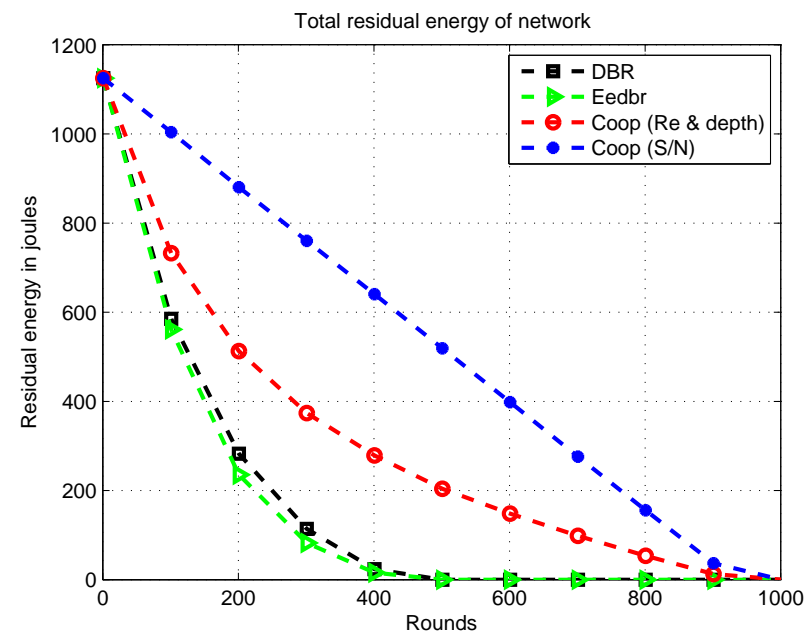

Figure 4.6: Comparison of total energy consumption of network with elliptical sink mobility pattern

DBR shows better energy management than EEDBR as shown in fig. 4.6. In DBR, residual energy decreases steadily as number of eligible neighbor drops off with network density. In EEDBR, energy consumption is higher than other techniques due to frequent selection of high energy nodes. In residual energy and $d_{t h}$ based cooperative criterion there is a sudden decrease in network's residual energy during the initial rounds as all nodes become active and perform the routing process. Later at, energy consumption decreases because nodes fail to find relay nodes due of reduction in network density. As a result, the chances of cooperative transmission being performed by any source node are reduced which in turn reduces energy consumption. SNR based criterion consumes almost constant energy throughout the network lifetime as compared to the other criterion because of proper forwarder node selection and implementation of well organized functions that involve depth difference and residual energy. Depth difference between data forwarders and their link state information give a better approximation of optimal data forwarder. 


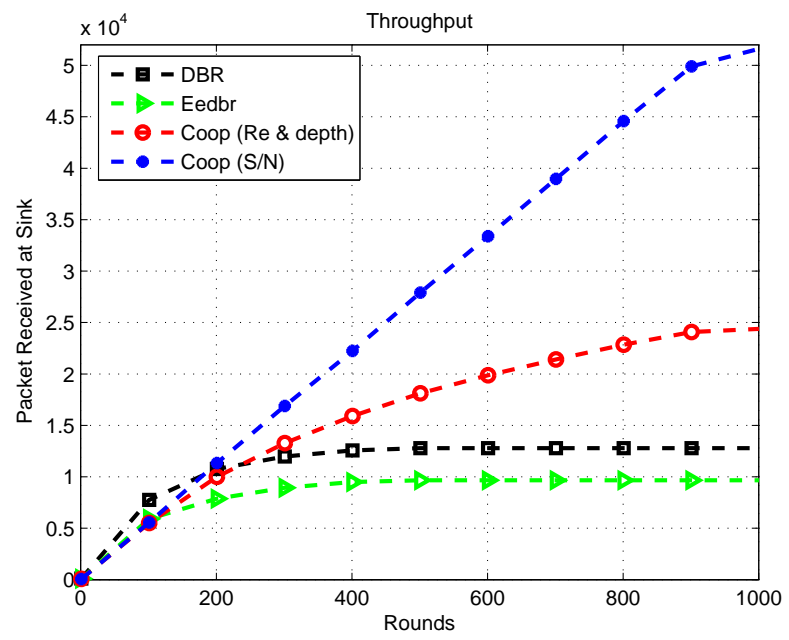

Figure 4.7: Comparison of packets received at MSs with elliptical sink mobility pattern

As shown in fig. 4.7, throughput of DBR and EEDBR declines very quickly due to the quick fall in network density, which is unsuitable for both delay-tolerant and delaysensitive applications. Throughput of our scheme demonstrates the amount of thresholdoptimized data for responsive network. In our scheme, unnecessary data transmissions are avoided that results in lower throughput as compared to DBR and EEDBR. In residual energy and $d_{t h}$ based cooperative criterion, throughput decreases sharply due to quick fall in network density. Whereas, in the other criterion, throughput decreases steadily due to maintenance of network density and low energy consumption.

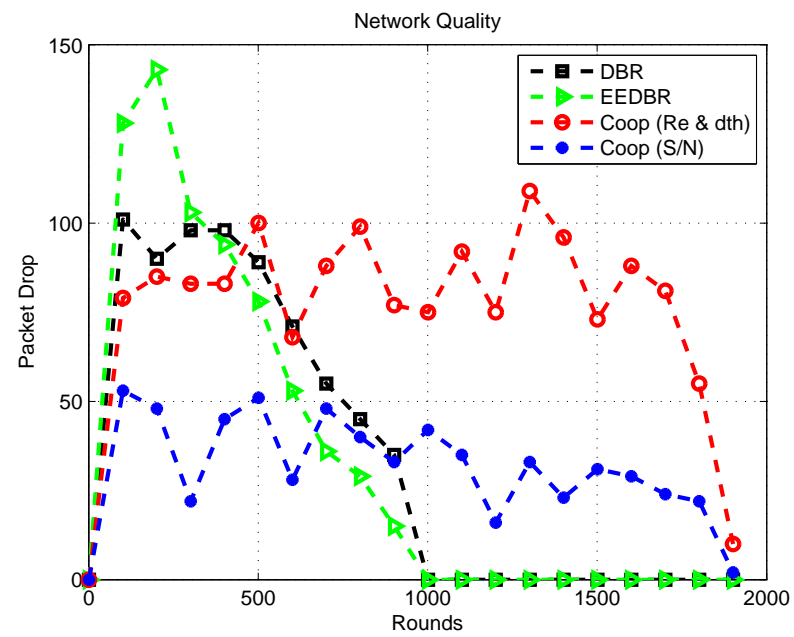

Figure 4.8: Comparison of packet drop with elliptical sink mobility pattern

Fig. 4.8 shows packet drop. High packet drop of DBR and EEDBR is due to single link having poor link state most of the time. Whenever the condition specified by the $H_{t h}$ and $S_{t h}$ is met, packets are generated by nodes. Sink only accepts those packets that possess an acceptable SNR and remaining are dropped. Plots show that residual energy 
and $d_{t h}$ based criterion gives higher packet drop because it does not take link quality into account. Whereas, SNR based criterion takes into account link quality in terms of SNR while selecting the forwarder node because of which best link is selected for data forwarding and packet drop is reduced.

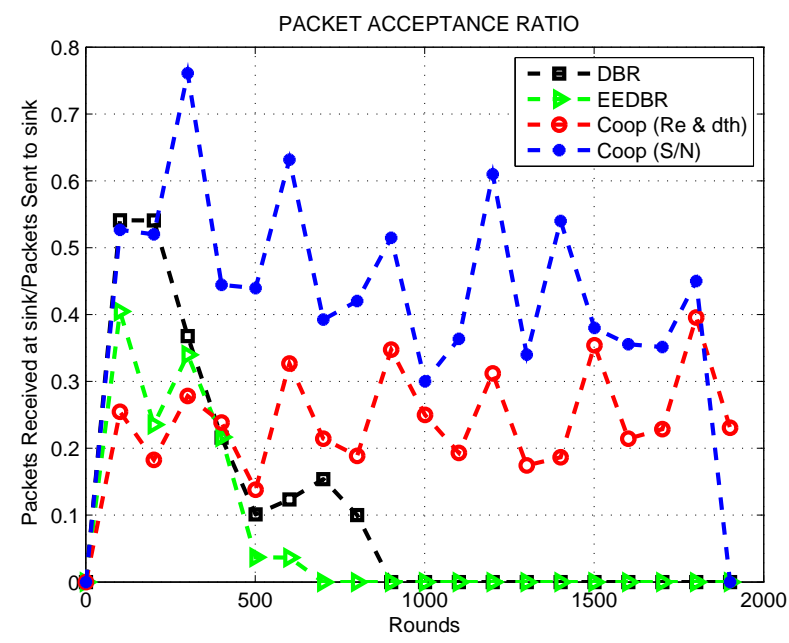

Figure 4.9: Comparison of PAR with elliptical sink mobility pattern

PAR is the ratio of packets received at sink and total number of packets generated by the nodes. It is shown in fig. 4.9. DBR and EEDBR have the lowest PAR as the number of packets received at sink in both these scenarios is small due to single link having poor link state most of the time. As packet drop is higher in the residual energy based criterion hence PAR is smaller, where the SNR based criterion yields higher PAR. 


\section{Chapter 5}

\section{DEADS: Depth and Energy $\underline{\text { Aware }}$ Dominating $\underline{\text { Set }}$ based Algorithm for Cooperative Routing along with Sink Mobility in Underwater WSNs}




\subsection{Motivation}

In UWSNs, high throughput along with reliability of the received information, reduced delay, low BER and energy conservation are of great concern. Novel routing solutions are therefore required for efficient data routing. Cooperative communication achieves high throughput along with reliable data transfer by reducing BER, however, it results in high propagation delay. Sink mobility reduces propagation delay and achieves energy conservation, however, it does not cater for reliability of received information. Many protocols involving cooperative communication are developed by investigating physical and MAC layer aspects, however, at network layer it is still unexplored. Similarly, the mechanism of implementing sink mobility along with cooperative routing at the network layer is still unattended. Therefore, this work focuses on an efficient mathematical model for cooperative routing along with sink mobility in UWSNs because we are interested in taking advantage of positive aspects of both the techniques along with minimizing their drawbacks.

\subsection{Channel Model}

Channel model is discussed in this section. Each source node modulates its data using Binary Phase Shift Keying (BPSK) modulation scheme. Channel over which this modulated data is transmitted suffers Rayleigh fading and Additive White Gaussian Noise (AWGN) noise. Equations (5.1-5.3) [26] describe relationship between the signals that are transmitted and received by source, relay and destination nodes. $X_{s}$ is the original signal, $Y_{s d}$ and $Y_{s r}$ are signals received at destination and relay nodes via source node. $Y_{r d}$ is signal received at destination node via relay node. $n_{s d}$ and $n_{s r}$ are channel noises existing over source to destination and source to relay links. $n_{r d}$ represents channel noise existing over relay to destination link. $g_{s d}$ and $g_{s r}$ are channel gains existing over source to destination and source to relay links. $g_{r d}$ represents channel gain existing over relay to destination link. Signals received at relay and destination nodes via source node are represented as:

$$
\begin{aligned}
& Y_{s r}=X_{s} g_{s r}+n_{s r} . \\
& Y_{s d}=X_{s} g_{s d}+n_{s d} .
\end{aligned}
$$


Signal received at destination node via relay node is represented as:

$$
Y_{r d}=Y_{s r} g_{r d}+n_{r d}
$$

Two independently faded copies $\left(Y_{s d}\right.$ and $\left.Y_{r d}\right)$ of source's data are combined at destination node by using a diversity combining technique.

\subsection{Mathematical Model}

In the proposed model, we consider that nodes are randomly deployed in an underwater environment, as shown in fig. 5.1. All nodes have a predefined transmission range associated with them. Initially, nodes do not possess depth and residual energy information of one-hop neighbors. This information is attained via broadcasting phase. In this phase, all nodes share depth and residual energy information with one-hop neighbors. Different rules and concepts that are followed in our algorithm are detailed:

Rule 1: Network is divided into 4 distinct regions or sub-graphs $\left(R_{k} \mid k=1-4\right)$ on the basis of depth $(D)$, such that, in any subgraph all nodes are one-hop apart and lie within each other's transmission range. Fig. 5.1 signifies rule 1 by showing one-hop apart nodes within each sub-graph.

$\mathrm{D}\left(R_{1}\right)>D\left(R_{2}\right)>\mathrm{D}\left(R_{3}\right)>\mathrm{D}\left(R_{4}\right)$

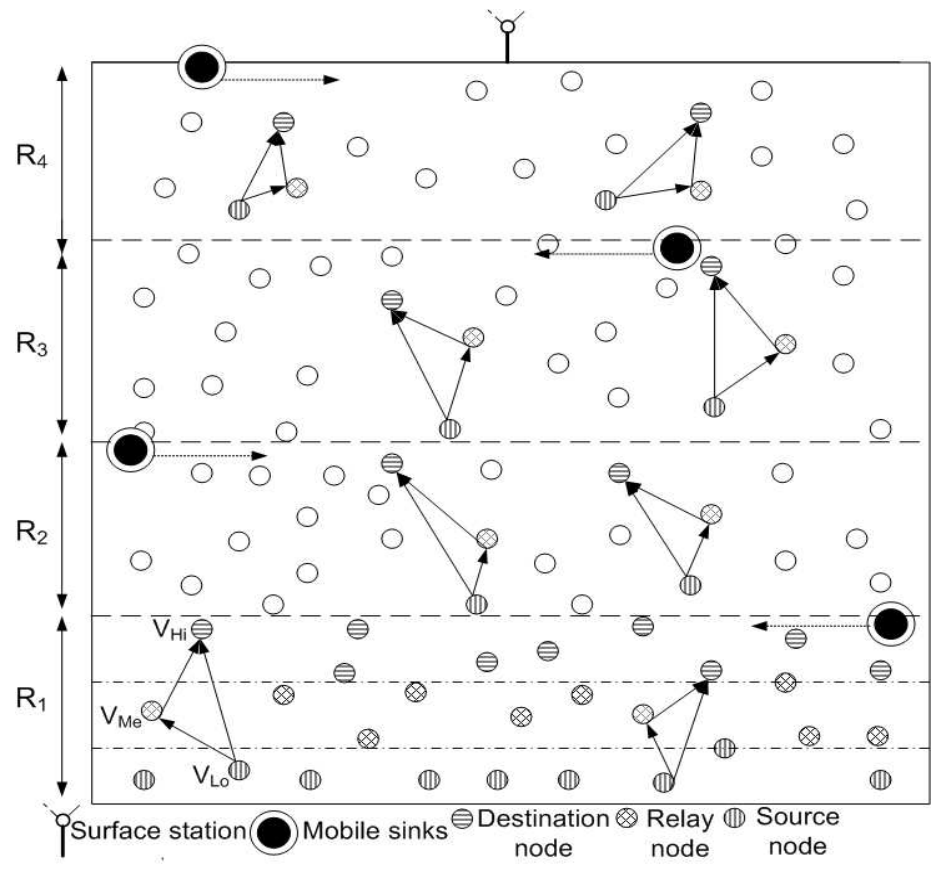

Figure 5.1: Network model

Rule 2: In each $R_{k}$, vertices are divided among three distinct sets, $V_{H i}, V_{M e}$ and $V_{L o}$ as 
shown in fig. 5.1, where

- $V_{H i}$ are high depth vertices in a sub-graph

- $V_{M e}$ are medium depth vertices in a sub-graph

- $V_{L o}$ are low depth vertices in a sub-graph

A $D S$ is formed for each $R_{k}$ on the basis of a certain criterion. We define $D S$ with respect to this work as;

$D S: D S$ is a subset of vertices $V$ of any $R_{k}$ such that every vertex not in the $D S$ is adjacent to at least one vertex in $D S$.

$D S$ for cooperative routing is computed on the bases of depth and residual energy information of sensor nodes. Nodes having lowest depth and highest residual energy in each $R_{k}$ form a $D S$ such that all the other nodes in that $R_{k}$ are connected to at least one node in $D S$.

Rule 3: Source, relay and destination nodes in any $R_{k}$ belong to $V_{L o}, V_{M e}$ and $V_{H i}$ such that;

$D_{D}<D_{R}<D_{S}$,

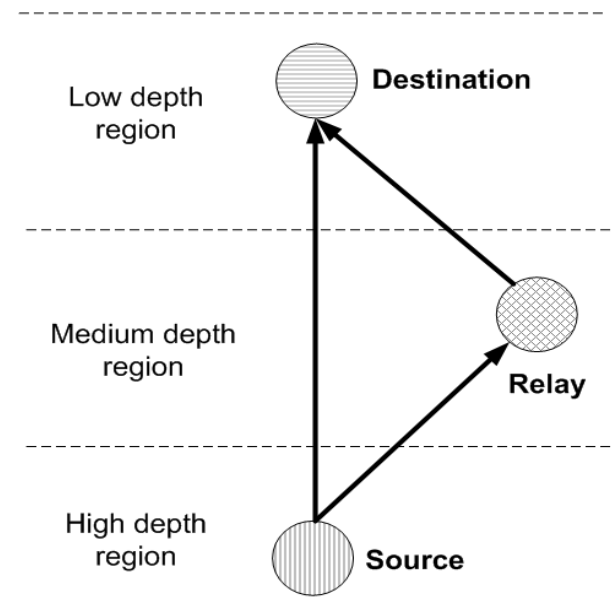

Figure 5.2: Rule 3: $D_{D}<D_{R}<D_{S}$

where $D_{D}, D_{R}$ and $D_{S}$ represent depth of destination, relay and source nodes respectively. This relation shows that source, relay and destination nodes are present at highest, medium and lowest depth regions in any $R_{k}$, respectively. Rule 3 is shown in fig. 5.2. We assume that MSs are periodically transmitting beacon signals because of which nodes are aware of their mobility pattern and are forwarding data accordingly.

Flooding is a routing process in which each incoming data packet is sent via every outgoing link except the one from which it was received. Flooding results in duplication of data 
and wastes resources by increasing burden on network. In depth-based routing, depth threshold $\left(d_{t h}\right)$ is used to avoid flooding by limiting the number of outgoing links for each incoming data packet.

Variations in $\mathbf{d}_{t h}$ : DBR [16], EEDBR [17] and iAMCTD [18] are existing depth-based routing protocols in UWSNs. In all these protocols, $d_{t h}$ is selected and varied according to the network density information which requires exchange of control packets at regular time interval. This exchange acts as an overhead and wastes network resources. We present a way for varying $d_{t h}$ that avoids this control packet exchange.

Rule 4: Value of $d_{t h}$ is selected and varied according to the number of a node's alive neighbors i.e.

$$
\forall i \in V: d_{t h_{i}}(t) \propto N_{i}(t)
$$

where, $N_{i}(\mathrm{t})$ represents alive neighbors of $i_{t h}$ node at time instant $t$

$$
N_{i}=N(i)=j \mid(i, j) \in A
$$

$\uparrow N \rightarrow \uparrow d_{t h}, \downarrow N \rightarrow \downarrow d_{t h}$

This relation signifies that more is the number of alive neighbors, higher is the value of $d_{t h}$ and vice versa. In this way, nodes just require one-hop neighbor information instead of complete network information. $d_{t h}$ plays an important role in selection of destination and relay nodes. Neighbors of a node are distinguished as potential $D S$ and Cooperative Connector $(C C)$ nodes on the basis of $d_{t h}$ as shown in fig. 5.3. Destination and relay nodes belong to $D S$ and $C C$ node sets.

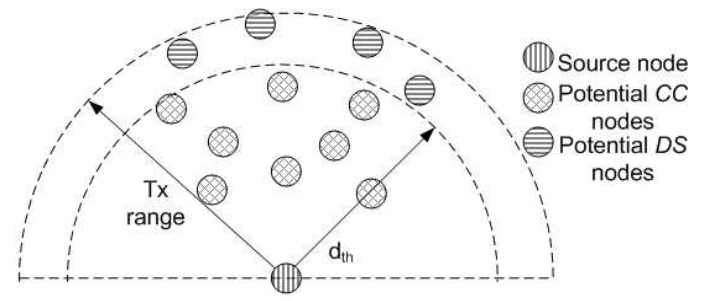

Figure 5.3: Depth threshold selection (highest $d_{t h}$ )

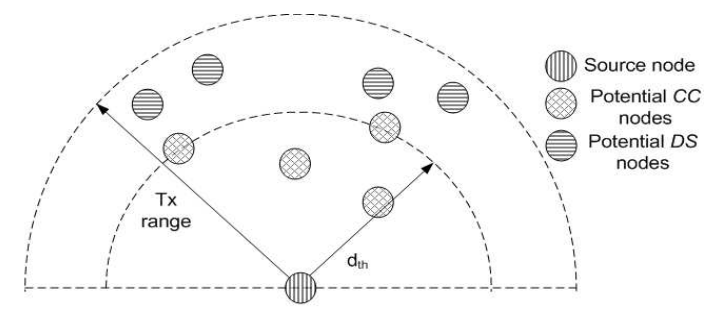

Figure 5.4: Depth threshold selection (medium $d_{t h}$ ) 


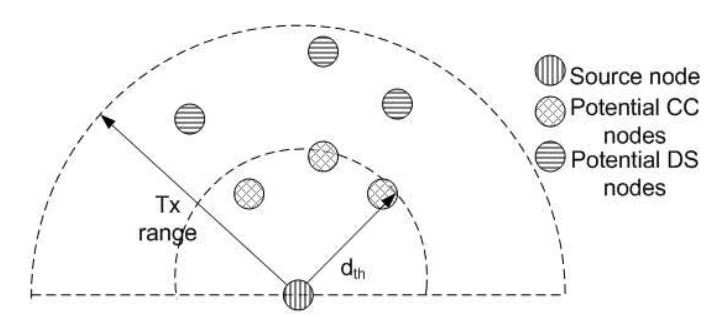

Figure 5.5: Depth threshold selection (lowest $d_{t h}$ )

As shown in fig. 5.3, number of nodes are present within the transmission range of source node. Due to this high node density its $d_{t h}$ is high $\left(d_{t h}^{h}\right)$. As node density within the transmission range decreases, $d_{t h}$ also decreases. $d_{t h}^{m}$ and $d_{t h}^{l}$ show the decreased $d_{t h}$ in fig. 5.4 and 5.5 .

Rule 5: Neighbor set of an $i_{t h}$ node is represented as

$$
N(i)=N_{\text {outd }_{t h}}(i) \cup N_{\text {ind }_{t h}}(i)
$$

where,

- $N_{\text {outd }_{t h}}(i)$ represents neighbors of an $i_{t h}$ node that lie outside the boundary specified by $d_{t h}$ as shown in fig. 5.3, 5.4 and 5.5 as potential $D S$ nodes.

- $N_{i n d_{t h}}(i)$ represents neighbors of an $i_{t h}$ node that lie inside the boundary specified by $d_{t h}$ as shown in fig. 5.3, 5.4 and 5.5 as potential $C C$ nodes.

$$
N_{\text {outd }_{t h}} \in V_{L o}, N_{\text {ind }_{\text {th }}} \in V_{M e}
$$

Eq. 5.7 signifies that $N_{\text {outd }_{t h}}(i)$ and $N_{i n d_{t h}}(i)$ are low and medium depth vertices in any $R_{k}$, respectively.

Rule 6: For each $R_{k}$, the potential $D S$ nodes are

$$
D S_{k} \subset N_{\text {outd }_{\text {th }}}\left(V_{H i}\right) \forall V_{H i} \in k \text {. }
$$

Initially, for any particular $R_{k}$, lowest depth one-hop neighbors $\left(N_{\text {outd }}\right.$ th $)$ of vertices represented by $V_{H i}$ are considered eligible for inclusion in the $D S$. Smaller the size of $D S$, lesser is the message overhead and network routing efficiency is also improved. In order to reduce the size of $D S$, nodes are further pruned, using their residual energy as a pruning parameter. After pruning in each $R_{k}$, lowest depth nodes possessing highest energy are present in $D S$.

Rule 7: The lowest depth and highest energy $D S$ nodes are the destination nodes for cooperative routing. Whereas, Probability of selection $(P s)$ of any node $i$ belonging to 
$V_{L o}$ as $D S$ node is

$$
\forall i \in V_{L o}:\left\{\begin{array}{l}
P s \propto R_{e}(i) \\
P s \propto 1 / D(i) .
\end{array}\right.
$$

Hence, Selection Parameter for $D S$ nodes, $\left(S P_{D S}\right)$, is a function of nodes' depth and residual energy:

$$
S P_{D S}=f(D, R e)
$$

$S P$ for $D S$ nodes at time $t, S P_{D S}(t)$ is given as:

$$
S P_{D S}(t)=R_{e}(t) / D(t),
$$

where,

$\mathbf{R}_{\mathbf{e}}(\mathbf{t})$ : Residual energy of any $D S$ node at time $t$ in joules

$\mathbf{D}(\mathbf{t})$ : Depth of any $D S$ node at time $t$ in meters

Higher the value of $S P_{D S}(t)$ for any particular node, more will be the chance of its inclusion in $D S$ and vice versa.

The highest energy one hop neighbors (belonging to $N_{i n d_{t h}}$ ) of vertices represented by $V_{H i}$ are considered eligible to be used as CCs. CCs are the relay nodes used in cooperative routing. They connect source nodes with the $D S$ nodes via an alternate path.

Rule 8: For each $R_{k}, C C$ nodes are:

$$
C C_{k} \subset N_{i n d_{t h}}\left(V_{H i}\right) \forall V_{H i} \in k .
$$

Rule 9: The highest energy nodes are the $C C s$ for cooperative routing. Where, $P s$ of any node $i$ belonging to $V_{M e}$ as $C C$ node is:

$$
\forall i \in V_{M e}: P s \propto R_{e}(i) .
$$

Hence, Selection Parameter for $C C$ nodes, $\left(S P_{C C}\right)$, is a function of nodes' residual energy:

$$
S P_{C C}=f\left(R_{e}\right) .
$$

$S P$ for the $C C$ at time $t$ is 


$$
S P_{C C}(t)=R_{e}(t) .
$$

Larger is the value of $S P_{C C}(t)$ for any particular node belonging to $V_{M e}$, more will be the chances of its inclusion in $C C$ node set and vice versa.

Rule 10: All $C C$ and non $-C C$ medium depth vertices in each $R_{k}$ forward their data towards non - DS low depth vertices directly or in a cooperative manner (depending on node availability).

$$
\begin{aligned}
& \forall i \in V_{M e}\left|i \in C C_{k}, R=j \in V_{M e}\right| j \notin C C_{k} \& D=l \in V_{L o} \mid l \notin D S_{k} . \\
& \forall i \in V_{M e}\left|i \notin C C_{k}, R=j \in V_{M e}\right| j \notin C C_{k} \& D=l \in V_{L o} \mid l \notin D S_{k} .
\end{aligned}
$$

Similarly, all non - DS low depth vertices in any sub-graph $k$ forward their data towards MSs directly or in a cooperative manner (depending on node availability). For any particular $R_{k}$;

$$
\begin{aligned}
& \forall i \in V_{L o}\left|i \in D S_{k}, D=M S \& R=j \in V_{L o}\right| j \notin D S_{k} . \\
& \forall i \in V_{L o}\left|i \notin D S_{k}, D=M S \& R=j \in V_{L o}\right| j \notin D S_{k} .
\end{aligned}
$$

Where, $D$ and $R$ specify destination and relay nodes. For this specific scenario, $D$ and $R$ are used instead of $D S$ and $C C$ due to the reason that $D$ and $R$ do not belong to $D S$ or $C C$ node set.

$D S$ recalculation is performed at regular time interval, $t$, in order to achieve balanced energy consumption throughout the network operation. Major advantage of $D S$ based routing is that it limits the routing process towards sink, mainly to the $D S$ nodes (according to rule 6 and 7 ). 


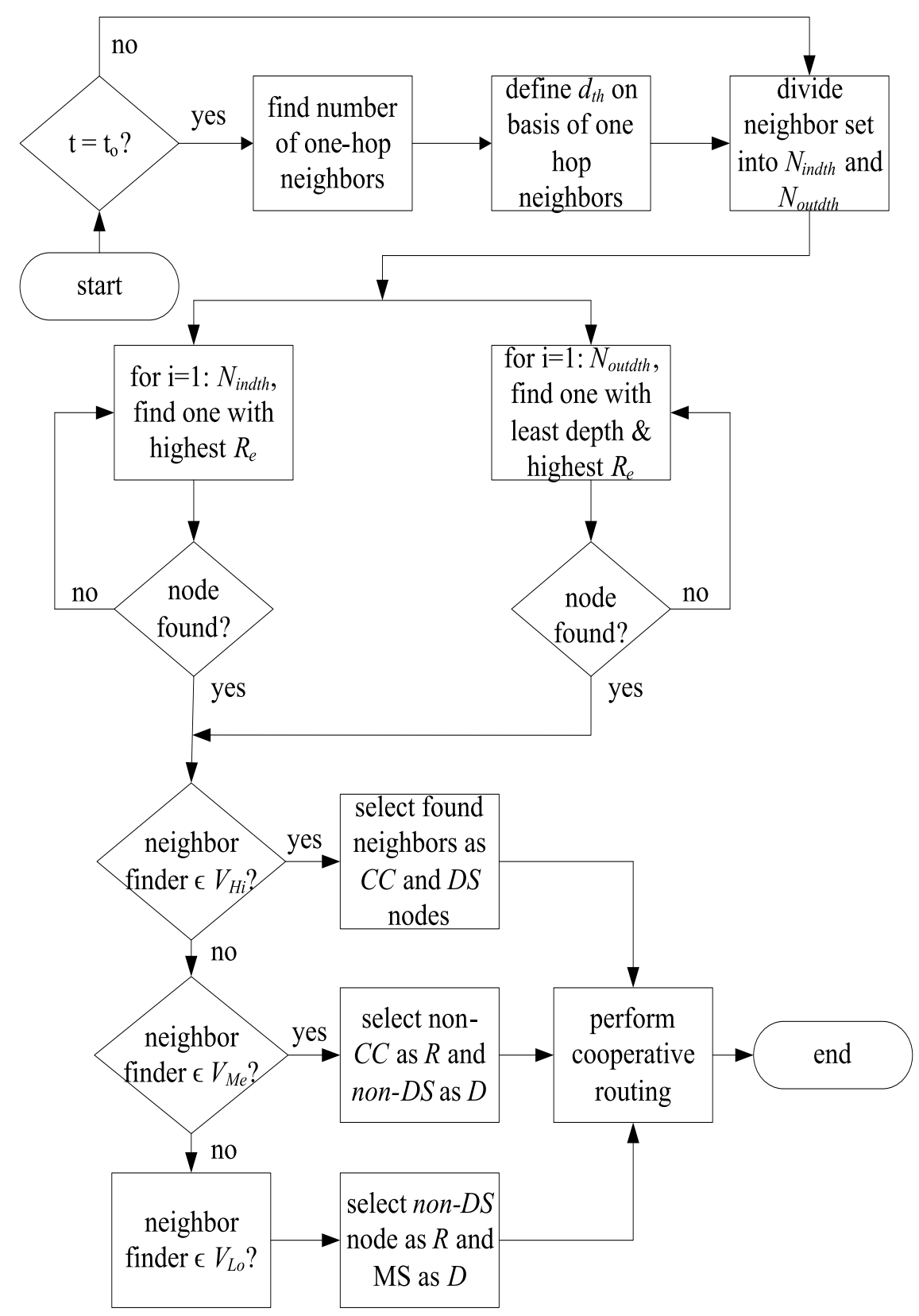

Figure 5.6: Flowchart showing selection of relay and destination nodes in a particular subgraph

Flow chart in fig. 5.6 shows the selection of relay and destination nodes which is then followed by cooperative routing. All nodes find their one-hop neighbors at regular time intervals, $t_{o}$ and define their $d_{t h}$ by following rule 4 . After selection of $d_{t h}$, neighboring nodes are differentiated among $N_{i n d_{t h}}$ and $N_{\text {outd }}$ th according to rule 5 . Nodes belonging to $V_{H i}$ use rule 6-9 for the selection of $C C$ and $D S$ nodes. $C C$ node belongs to $N_{\text {indth }}$ and possesses the highest $R_{e}$, whereas, destination node is one that belongs to $N_{\text {out }} d_{\text {th }}$ and possesses the highest $R_{e}$ and least depth. Nodes belonging to $V_{M e}$ and $V_{L o}$ follow rule 10 for selection of relay and destination nodes. If the neighbor finder node belongs to $V_{M e}$ of any particular sub-graph, then it selects any non $-C C$ node as a relay and 
non - DS node as a destination node. On the other hand, if the neighbor finder node belongs to $V_{L o}$ of any particular sub-graph, then it selects any non - DS node as a relay, MS as a destination node and forwards data cooperatively.

\subsection{DEADS: The Proposed Scheme}

Detailed discussion of the proposed protocol (DEADS) is given in the following subsection. Data sensing and routing example explains the selection of relay and destination nodes in a sub-graph.

\subsubsection{Data Sensing and Routing}

Let us consider a particular sub-graph as shown in fig. 5.7 to describe the construction of $D S$. In this example, depth and energy aware $D S$ construction for a particular subgraph is shown. For any vertex $a$, we represent its neighbor set as; $N(a)=b \in V \mid a, b \in A$ where $b \in n$ ( $n$ represents total number of nodes in a network).

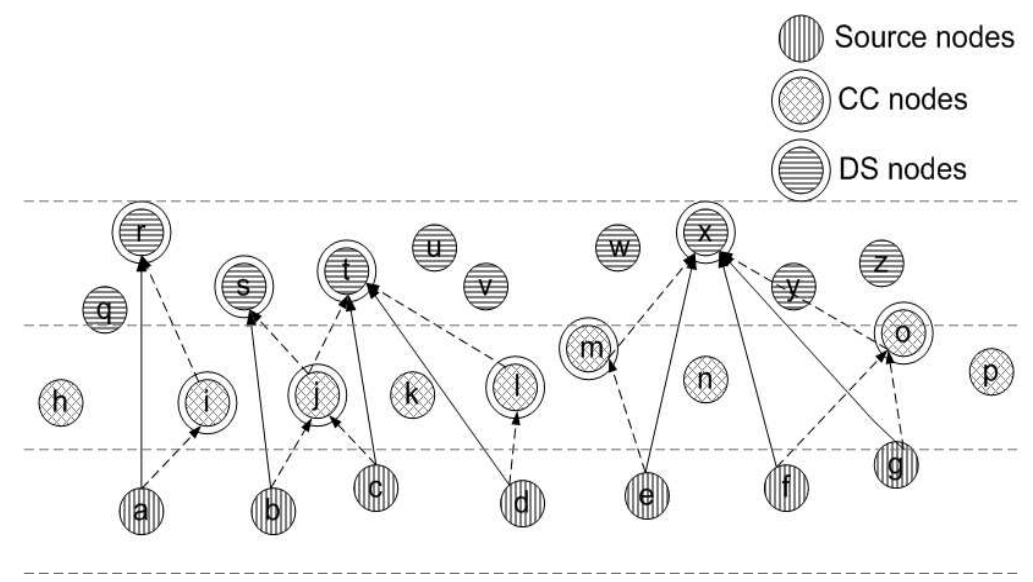

Figure 5.7: Selection of DS and CC nodes in a sub-graph

Initially, each source node finds the number of its one-hop neighbors and selects $d_{t h}$ on its basis. $d_{t h}$ selection process in shown in algorithm 1 . Network lifetime is represented in terms of seconds. $d_{t h}$ recalculation is performed at regular intervals.

Once a node becomes aware of its $d_{t h}$, it divides its neighbor set into two distinct sets $\left(N_{\text {outd }_{t h}}\right.$ and $\left.N_{\text {ind }_{t h}}\right)$ as shown by the given expressions. In this sub-graph, eligible $D S$ nodes are $N_{\text {outd }}$ th and the eligible $C C$ nodes are $N_{i n d_{t h}}$. For example, node $a$ has nodes $h, i, j, q, r$ and $s$ within its transmission range. After finding its $d_{t h}$ node $a$ divides its neighbor set into two sets, finds $C C$ and $D S$ nodes by following rule 6 to 9 and perform cooperative routing. Node $b, c, d, e, f$ and $g$ follow the same procedure as followed by 


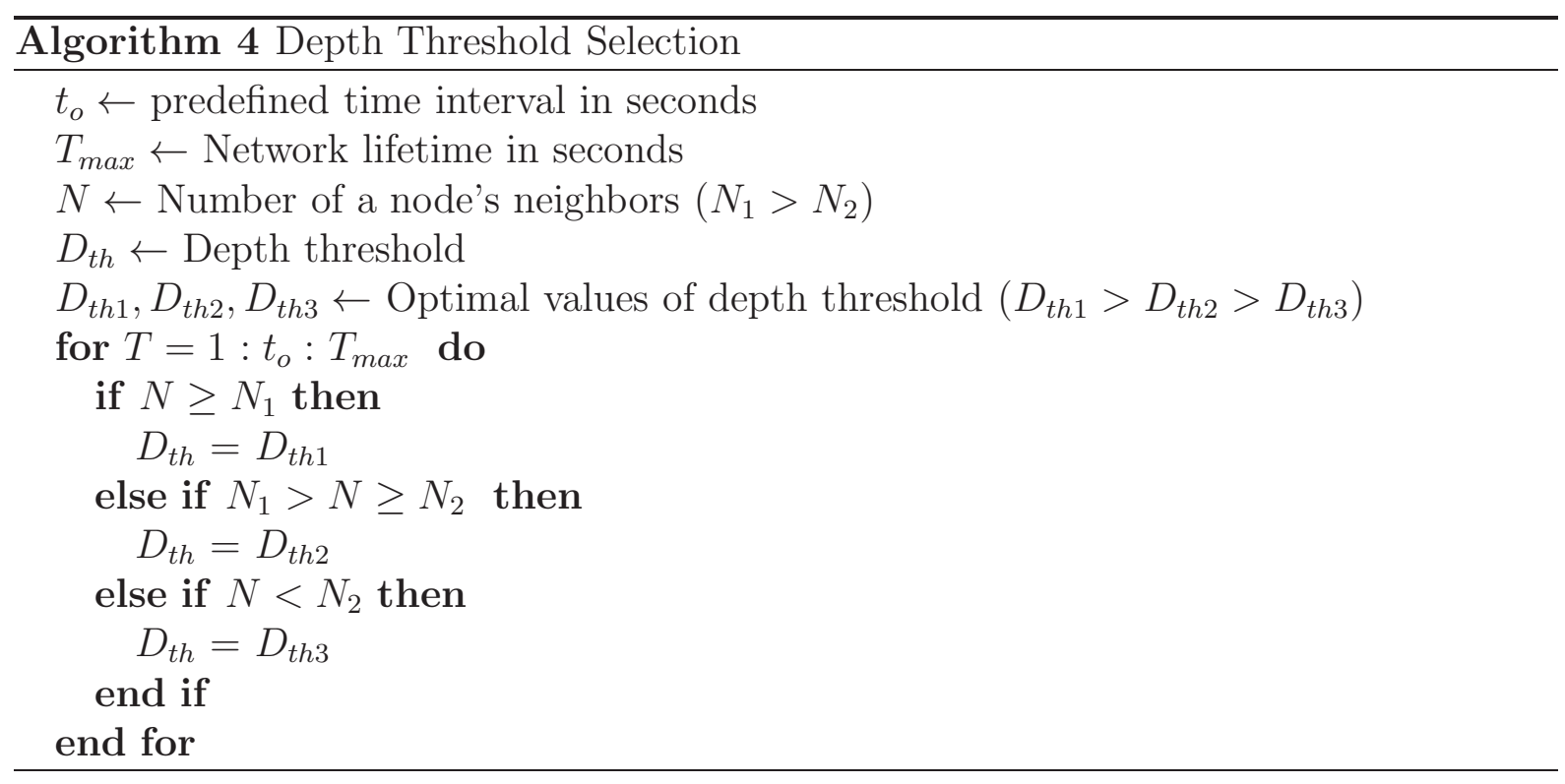

node $a$. Whereas, all the non $-C C$ and non $-D S$ nodes forward data by following rule 10.

$$
\begin{aligned}
& N(a)=h, i, j, q, r, s, N_{\text {ind }_{t h}}(a)=h, i, j, N_{\text {outd }_{t h}}(a)=q, r, s, \\
& N(b)=i, j, k, r, s, t, N_{\text {ind }_{t h}}(b)=r, e, h, i, N_{\text {outd }_{t h}}(b)=r, s, t, \\
& N(c)=j, k, i, l, s, t, u, v, N_{i n d_{t h}}(c)=j, k, i, l, N_{\text {outd }_{t h}}(c)=s, t, u, v, \\
& N(d)=k, l, m, t, u, v, N_{\text {ind }_{t h}}(d)=k, l, m, N_{\text {outd }_{t h}}(d)=t, u, v, \\
& N(e)=l, m, n, w, x, y, N_{\text {ind }}(e)=l, m, n, N_{\text {outd }}(e)=w, x, y, \\
& N(f)=m, n, o, w, x, y, z, N_{\text {ind }_{t h}}(f)=m, n, o, N_{\text {outd }_{\text {th }}}(f)=w, x, y, z, \\
& N(g)=n, p, o, x, y, z, N_{i n d_{t h}}(g)=p, n, o, N_{\text {outd }_{t h}}(g)=x, y, z,
\end{aligned}
$$

Traditional routing schemes [16], [17] consume surplus energy due to transmission of redundant data and retransmission of critical data. We exploit threshold based data sensing in which data is transmitted only when certain predefined thresholds are met. Threshold values depend on the required application. Algorithm 2 describes data sensing and routing procedure. After nodes become aware of their neighbors, they start sensing the specified environmental attribute and forward the sensed data by using cooperative routing.

According to rule 6 (eq. 5.8), $D S$ is formed and by utilizing $S P_{D S}$ given in rule 7 (eq. 5.9-5.11) each node selects an appropriate destination node that belongs to the $D S$ nodes. Similarly, according to rule 8 (eq. 5.12), $C C$ set is formed and by utilizing rule 9 (eq.5.135.15) each node selects an appropriate relay node that belongs to the $C C$ nodes. $C C$, non - $C C, D S$ and non - DS nodes also forward their data by utilizing rule 10 (eq. 5.165.19). In this way, the most optimal destination and relay nodes are selected cooperative 


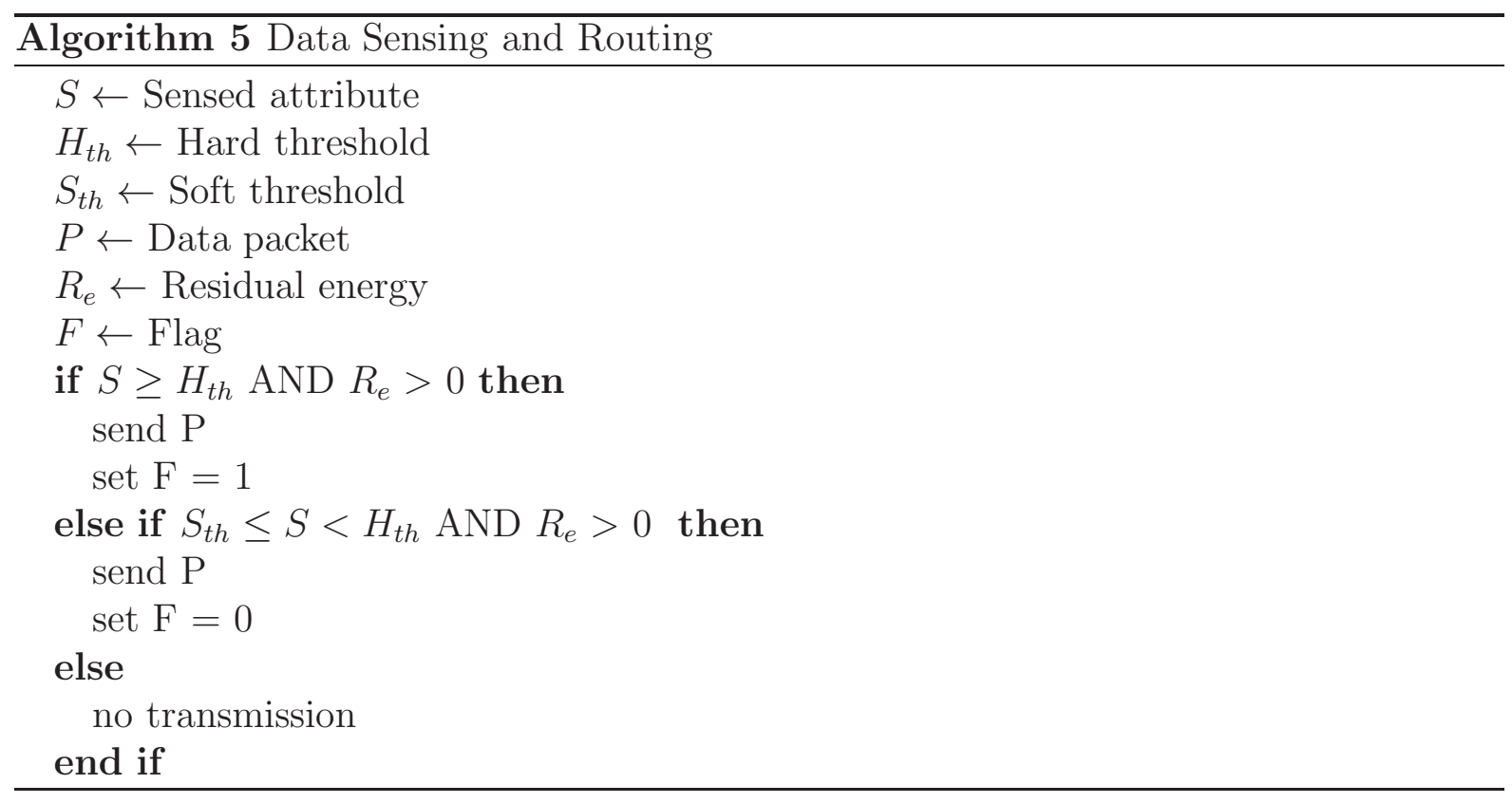

routing as shown in fig. 5.7. $D S$ nodes and non $-D S$ destination nodes implement Maximal Ratio Combining (MRC) as a diversity combining technique on received copies of the data and forward it to MS in its vicinity.

\subsubsection{Sink Mobility}

Two different sink mobility patterns are used in this work. These mobility patterns are discussed in detail in the following subsection.

\subsubsection{Linear Mobility Pattern}

MSs follow a horizontally varying linear mobility pattern. MSs are present at different depths $\left(y_{0}\right)$ that remain same throughout the network lifetime. They move either leftwards or rightwards to cover the entire network. MSs change their position after predefined time interval. New position of MSs after a predefined time interval is represented as:

$$
\begin{gathered}
x_{M S_{L}}=x_{o}-n_{o} . \\
x_{M S_{R}}=x_{o}+n_{o} . \\
y_{M S_{L}}=y_{M S_{R}}=y_{o} .
\end{gathered}
$$

Where eq. 5.20, 5.21 and 5.22 indicate $x$ and $y$ dimensions of MSs that are moving leftwards or rightwards. $n_{o}$ represents horizontal shift at each predefined time interval 
and its value can be adjusted according to network requirements. Fig. 5.8 shows data routing in the network involving linear sink mobility pattern. Data is routed from high depth to low depth region according to the rules defined in section IV. After finding $d_{t h}$, nodes find appropriate $C C$ or $R, D S$ or $D$ nodes and then forward data in a cooperative manner. Nodes forward data by using either Single Relay Communications (DEADS$\mathrm{SRC}$ ) or Multiple Relay Communications (DEADS-MRC, two relay nodes in our case). As shown in fig. 5.8, node $A$ and $B$ are forwarding data towards the preselected next-hop $(D S$ or $D)$ node via MRC, whereas, node $C$ forwards data via SRC.

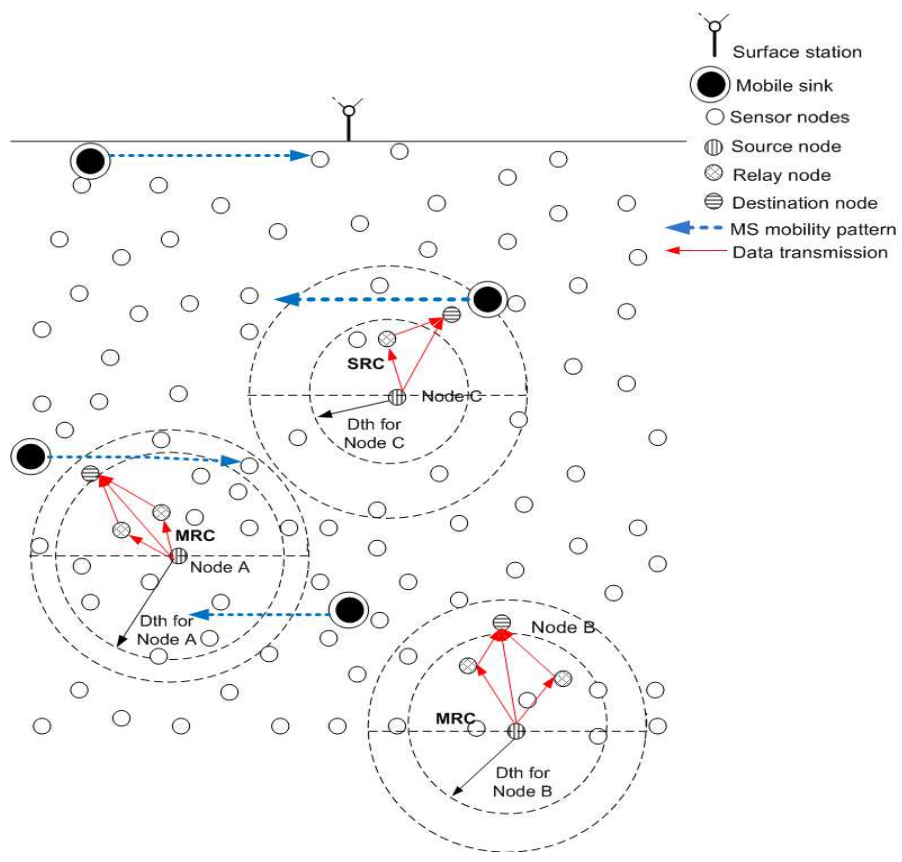

Figure 5.8: Data routing in the network with linear sink mobility pattern

\subsubsection{Elliptical Mobility Pattern}

Each MS follow an elliptical mobility pattern (with vertical major-axis) represented by equation:

$$
(x / b)^{2}+(y / a)^{2}=1
$$

MSs move either Clock-Wise $(C W)$ or Counter Clock-Wise $(C C W)$ to cover the entire network. MSs change their position after regular time interval. New position of MSs represented by their $x$ and $y$ dimensions is given by:

$$
\begin{aligned}
& x_{M S_{C W, C C W}}=x_{o}+a \times \cos (\theta) . \\
& y_{M S_{C W, C C W}}=y_{o}+b \times \sin (\theta) .
\end{aligned}
$$




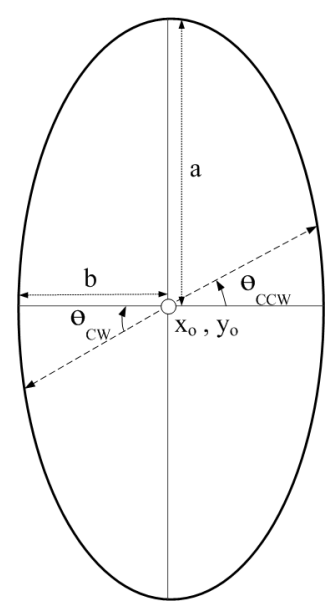

Figure 5.9: Specifications of elliptical mobility path followed by MSs

Fig. 5.9 shows the elliptical mobility path followed by each MS. $x_{o}$ and $y_{o}$ represent the center coordinates of each elliptical path which are the same for each ellipse. $a$ and $b$ represent semi-major and semi-minor axis of each elliptical pattern, where; $a=2 b$. Values of $a$ and $b$ are different for each elliptical path. $\theta$ represents the angle which is varied for clock-wise or counter clock-wise movement. After each predefined time interval, $\theta$ is incremented or decremented by a certain value for counter clock-wise or clock-wise movement of MSs. Value of $\theta$ can be adjusted according to the network requirements. Fig. 5.10 shows data routing in the network in which MSs follow an elliptical mobility pattern. Data is routed from high depth to low depth region according to the rules defined in section IV. All the nodes find $d_{t h}$, find appropriate $C C$ or $R, D S$ or $D$ nodes and then forward data by using cooperative manner. Nodes forward data by using either DEADS-SRC or DEADS-MRC. As shown in fig. 5.10, node $D$ and $F$ are forwarding data towards the preselected next-hop $(D S$ or $D)$ node via MRC, whereas, node $E$ forwards data directly towards MS via SRC. 


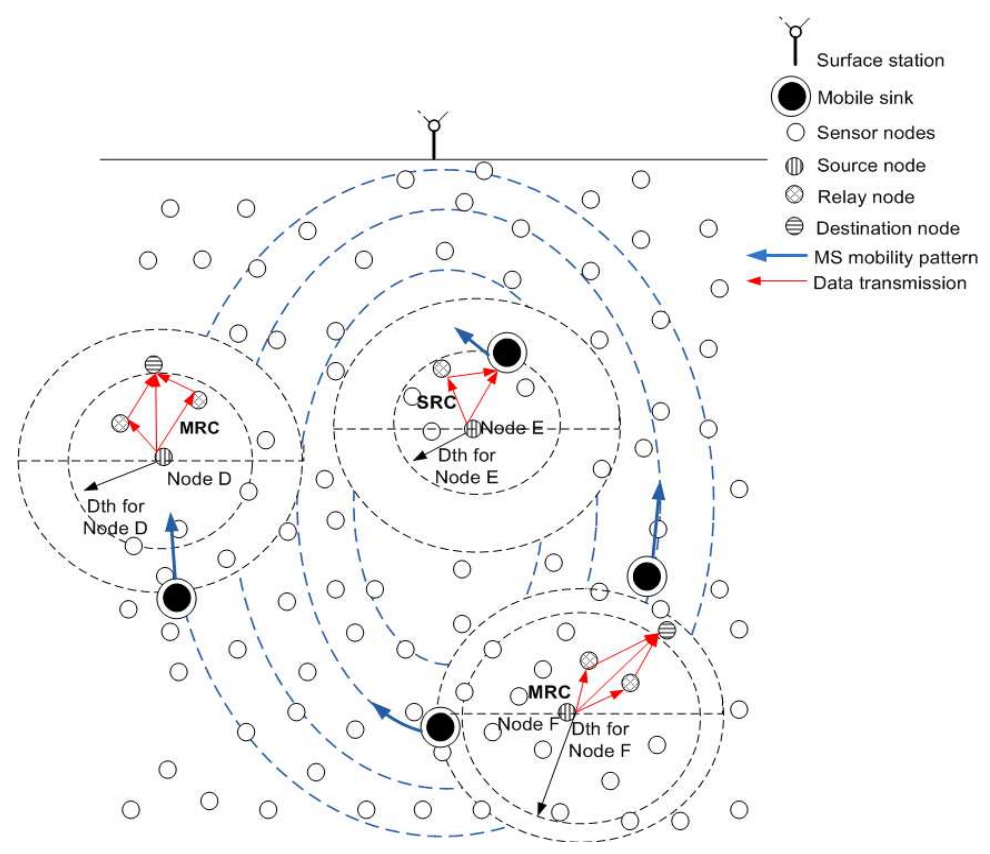

Figure 5.10: Data routing in the network with elliptical sink mobility pattern

\subsection{Simulation Results and Discussions}

We validate and evaluate our proposed scheme via simulation. We compare DEADSSRC and DEADS-MRC with existing depth-based routing protocols; DBR, EEDBR and iAMCTD. There are 225 sensor nodes which are randomly deployed in underwater environment at an area of $500 \mathrm{~m} \times 500 \mathrm{~m} \times 500 \mathrm{~m}$. Four MSs are deployed under water at different depths and they are following linear and elliptical mobility patterns. Each sensor node is equipped with an initial energy of 5 Joules and is having a fixed transmission range of $100 \mathrm{~m}$. Size of data packet and control packet is 200 byte and 8 byte respectively. LinkQuest UWM1000 [25] acoustic modem is used having $10 \mathrm{kbps}$ bit rate. Power consumption of a node in transmitting, receiving and in idle modes is $2 \mathrm{~W}, 0.1 \mathrm{~W}$ and $10 \mathrm{~mW}$, respectively. In iAMCTD, there are 4 static sinks and 4 courier nodes for data reception, however, in the proposed protocol only $4 \mathrm{MSs}$ are present. We excluded 4 static sinks present in iAMCTD in order to make it comparable with our proposed protocol.

DBR, EEDBR and CoDBR are proactive routing (continuous data routing) protocols, whereas, iAMCTD and DEADS are reactive routing (data routing occurs only when certain thresholds are met) protocols. An analysis of the proposed and existing protocols with respect to different parameters is given in table 5.1. DBR and CoDBR consider depth, EEDBR considers depth and residual energy and AAMCTD considers link's state in terms of SNR, depth and residual energy of the forwarding nodes as an ultimate forwarding node determining factor. Whereas, in DEADS, depth and residual energy of 


\begin{tabular}{|l|l|l|l|l|}
\hline Protocol & $\begin{array}{l}\text { Depth threshold } \\
\left(\mathbf{d}_{t h}\right)\end{array}$ & Sink mobility & Routing & $\begin{array}{l}\text { Parameter for for- } \\
\text { warder node selec- } \\
\text { tion }\end{array}$ \\
\hline DBR & Fixed & $\begin{array}{l}\text { Static sinks (on } \\
\text { water surface) }\end{array}$ & $\begin{array}{l}\text { Multi-hop } \\
\text { routing }\end{array}$ & Depth \\
\hline EEDBR & Fixed & $\begin{array}{l}\text { Static sinks (on } \\
\text { water surface) }\end{array}$ & $\begin{array}{l}\text { Multi-hop } \\
\text { routing }\end{array}$ & $\begin{array}{l}\text { Depth and residual } \\
\text { energy }\end{array}$ \\
\hline CoDBR & Fixed & $\begin{array}{l}\text { Static sinks (on } \\
\text { water surface) }\end{array}$ & $\begin{array}{l}\text { Cooperative } \\
\text { routing }\end{array}$ & Depth \\
\hline $\begin{array}{l}\text { DEACTD } \\
\text { (proposed) }\end{array}$ & $\begin{array}{l}\text { of network density } \\
\text { information) }\end{array}$ & $\begin{array}{l}\text { Mobile courier } \\
\text { nodes } \\
\text { information) }\end{array}$ & $\begin{array}{l}\text { Multi-hop } \\
\text { routing }\end{array}$ & $\begin{array}{l}\text { Depth, residual en- } \\
\text { ergy and link's SNR }\end{array}$ \\
\hline
\end{tabular}

Table 5.1: Analysis of existing depth-based routing protocols

the forwarding nodes are considered as forwarding node determining factor. To evaluate the performance of DEADS following evaluation metrics are considered:

Network lifetime: Network lifetime is the duration up till which all nodes in the network run out of energy. At any time instant, alive nodes represent the number of nodes which have sufficient residual energy for data transmission. Network lifetime increases when a balanced energy consumption is involved in the network. Presence of MSs for data collection also improves network lifetime by directly collecting data from nodes. Moreover, energy is conserved by avoiding flooding in the network which in turn increases network lifetime.

Residual energy: It is the total unexpended energy possessed by the network per time instant. Energy is conserved by avoiding flooding and by introducing sink mobility in the network. Sink mobility conserves energy by avoiding multi-hop transmission via directly gathering data packets from source nodes.

Throughput: It is the total number of data packets received at sink per unit time. Throughput can be increased by selecting optimal forwarder node having best link state at any instant. Short distance transmissions also increase throughput. Shorter the distance between two nodes, lesser will be the chances of data being corrupted by link impairments and consequently higher will be the throughput. Implementation of MRC as a diversity combining technique at next hop node also increases throughput.

Packet drop: It shows the number of dropped packets out of total number of packets generated by nodes. If link state is not taken into account while selecting forwarder nodes then chances of packet drop are high. Long distance transmission also increases packet 
drop.

Packet Acceptance Ratio (PAR): It is defined as the ratio of total no. of packets received at sink to the total no. of packets sent to the sink at regular time interval. Higher is the throughput more will be the PAR and vice versa.

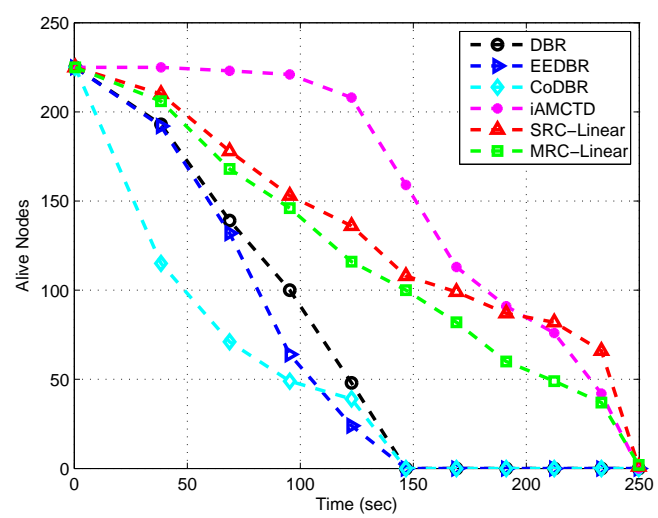

Figure 5.11: Comparison of alive nodes in the network with linear sink mobility pattern

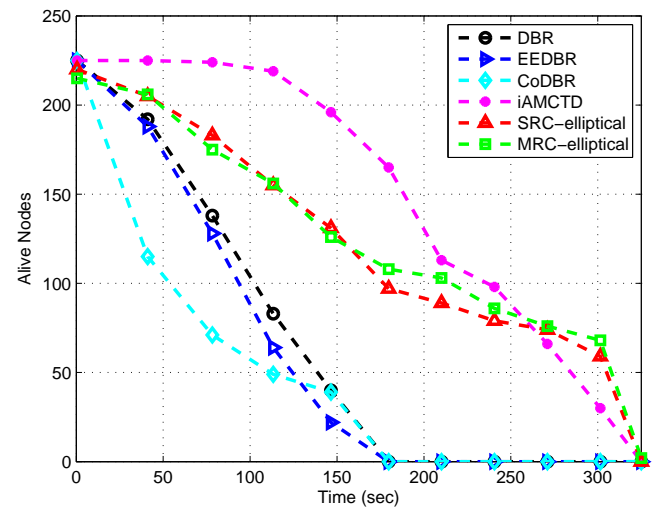

Figure 5.12: Comparison of alive nodes in the network with elliptical sink mobility pattern

As shown in fig. 5.11, 5.12 and table 5.2, in DBR, EEDBR and CoDBR, node density decreases sharply with time. The instability period is better in DBR as compared to EEDBR as there is a gradual increase in energy consumption. When network becomes sparse, number of neighbors decreases quickly which causes network instability. In DBR and CoDBR, low-depth nodes die at an earlier stage due to huge data forwarding and constant $d_{t h}$. DBR and CoDBR neglects both residual energy of nodes as well as link state in terms of SNR, whereas, EEDBR neglects link state which badly affects network throughput of these protocols. Number of dead nodes sharply increases with time in EEDBR as there is high load on high energy nodes because of considering residual energy as a routing metric. In CoDBR, network density decreases at the fastest rate 
because of cooperative routing being performed. Cooperative routing involves high energy consumption because of multiple transmissions and receptions of same data packet. Stability period (time up till which all nodes in the network are alive) of iAMCTD is highest because of optimal $d_{t h}$ selection. Also, in iAMCTD, only source node transmits data to its next hop neighbor which conserves energy and results in improved stability period. Although due to implementation of rule $4, d_{t h}$ selection is more optimized in DEADS-SRC and DEADS-MRC as it requires only one-hop neighbor information instead of complete network density information, however, data is transmitted from source node as well as relay node(s) to the next hop. So, it is consuming 2 (SRC) to 3 (MRC) times more transmission and reception energy than iAMCTD due to which stability period is compromised. This shows the tradeoff between network stability period and reliability.

\begin{tabular}{|l|l|l|l|l|l|l|l|}
\hline Protocol & $\mathbf{5 0}$ sec & $\mathbf{1 0 0} \mathbf{~ s e c}$ & $\mathbf{1 5 0} \mathbf{~ s e c}$ & $\mathbf{2 0 0} \mathbf{~ s e c}$ & $\mathbf{2 5 0} \mathbf{~ s e c}$ & $\mathbf{3 0 0}$ sec & $\mathbf{3 5 0}$ sec \\
\hline DBR & 170 & 90 & 0 & 0 & 0 & 0 & 0 \\
\hline EEDBR & 167 & 60 & 0 & 0 & 0 & 0 & 0 \\
\hline CoDBR & 100 & 50 & 0 & 0 & 0 & 0 & 0 \\
\hline iAMCTD & 225 & 220 & 152 & 85 & 0 & 0 & 0 \\
\hline $\begin{array}{l}\text { DEADS-SRC } \\
\text { (linear) }\end{array}$ & 198 & 150 & 108 & 83 & 0 & 0 & 0 \\
\hline $\begin{array}{l}\text { DEADS-SRC } \\
\text { (Elliptical) }\end{array}$ & 200 & 163 & 130 & 90 & 77 & 60 & 0 \\
\hline $\begin{array}{l}\text { DEADS-MRC } \\
\text { (linear) }\end{array}$ & 192 & 140 & 100 & 57 & 0 & 0 & 0 \\
\hline $\begin{array}{l}\text { DEADS-MRC } \\
\text { (Elliptical) }\end{array}$ & 200 & 160 & 125 & 105 & 80 & 65 & 0 \\
\hline
\end{tabular}

Table 5.2: No. of alive nodes after equal time intervals (sec)

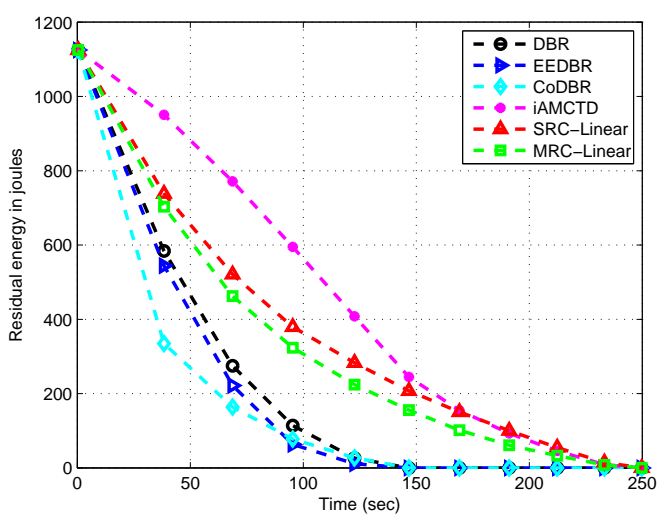

Figure 5.13: Comparison of total energy consumption of network with linear sink mobility pattern 


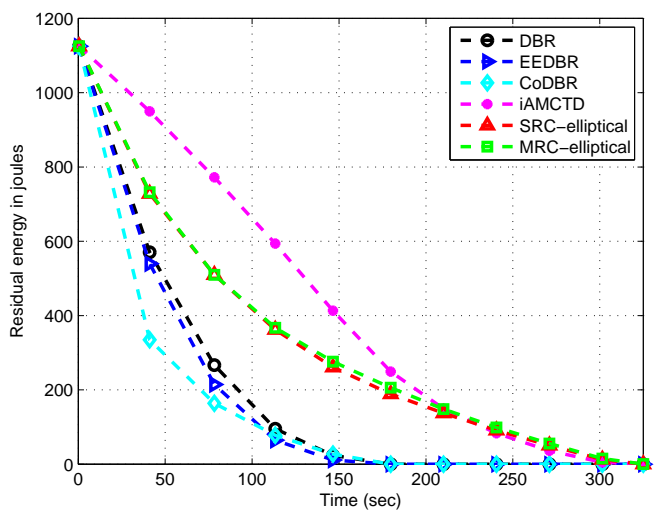

Figure 5.14: Comparison of total energy consumption of network with elliptical sink mobility pattern

DBR shows better energy management than EEDBR as shown in fig. 5.13, 5.14 and table 5.3. In DBR, network residual energy steadily decreases as total number of eligible neighbors drops off with network density. Energy consumption of EEDBR is the highest among all other protocols due to frequent selection of high energy nodes. Moreover, higher energy consumption in DBR, EEDBR and CoDBR is because of reactive routing being performed in these protocols. Although CoDBR attains data reliability and high throughput, however, energy consumption is highest because of cooperative routing in the network as well as flooding resulting from fixed $d_{t h}$. iAMCTD attains a balanced residual energy throughout the network lifetime due to proper forwarder selection, optimal mobility patterns of courier nodes and maintenance of uniform network density. In DEADS-SRC and DEADS-MRC, high energy consumption is involved as compared to iAMCTD. This high energy consumption is because of cooperative routing. In the proposed protocol, as the network starts, nodes become active and start the sensing and transmission process. Initially all nodes are alive, each node finds appropriate relay and destination nodes and performs transmission and reception process due to which energy consumption is increased. After certain time, rate at which nodes become dead increases and energy consumption decreases. The reason behind this decreased energy consumption with increasing time is that nodes fail to find optimal relay nodes because of reduction in network density. As a result, chances of cooperative routing being performed by any source node are reduced which reduces energy consumption and most of the data is directly transmitted to the MSs or next hop node only. Table 5.3 shows that the proposed scheme achieves a much better energy management in spite of performing cooperative routing which involves high energy consumption. 


\begin{tabular}{|l|l|l|l|l|l|l|l|}
\hline Protocol & $\mathbf{5 0} \mathbf{~ s e c}$ & $\mathbf{1 0 0} \mathbf{~ s e c}$ & $\mathbf{1 5 0} \mathbf{~ s e c}$ & $\mathbf{2 0 0} \mathbf{~ s e c}$ & $\mathbf{2 5 0} \mathbf{~ s e c}$ & $\mathbf{3 0 0} \mathbf{~ s e c}$ & $\mathbf{3 5 0} \mathbf{~ s e c}$ \\
\hline DBR & 460 & 100 & 0 & 0 & 0 & 0 & 0 \\
\hline EEDBR & 400 & 56 & 0 & 0 & 0 & 0 & 0 \\
\hline CoDBR & 270 & 65 & 0 & 0 & 0 & 0 & 0 \\
\hline iAMCTD & 880 & 580 & 230 & 80 & 0 & 0 & 0 \\
\hline $\begin{array}{l}\text { DEADS-SRC } \\
\text { (linear) }\end{array}$ & 660 & 375 & 200 & 80 & 0 & 0 & 0 \\
\hline $\begin{array}{l}\text { DEADS-SRC } \\
\text { (Elliptical) }\end{array}$ & 680 & 405 & 260 & 170 & 18.5 & 10 & 0 \\
\hline $\begin{array}{l}\text { DEADS-MRC } \\
\text { (linear) }\end{array}$ & 605 & 320 & 170 & 60 & 0 & 0 & 0 \\
\hline $\begin{array}{l}\text { DEADS-MRC } \\
\text { (Elliptical) }\end{array}$ & 680 & 405 & 265 & 175 & 85 & 10 & 0 \\
\hline
\end{tabular}

Table 5.3: Network's residual energy in joules after equal time intervals (sec)

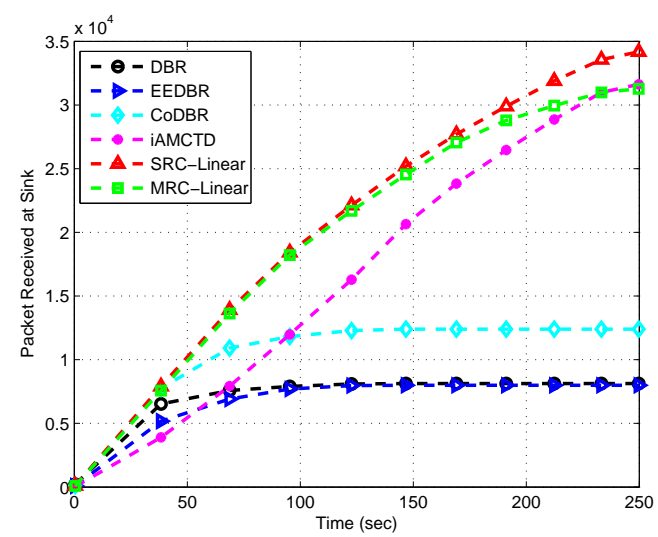

Figure 5.15: Comparison of packets received at MSs with linear sink mobility pattern

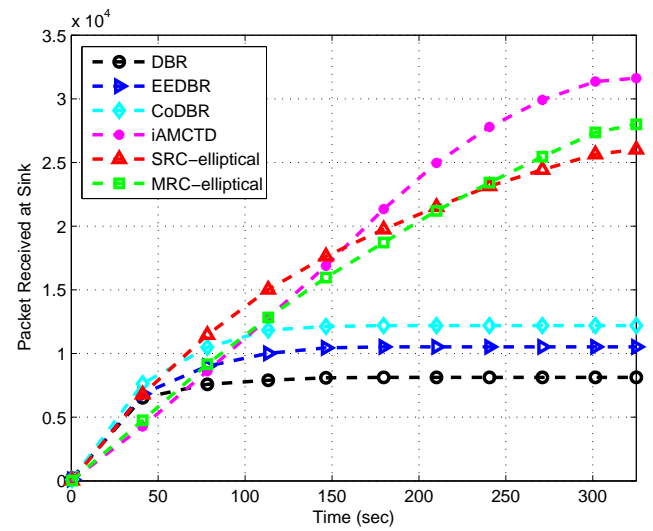

Figure 5.16: Comparison of packets received at MSs with elliptical sink mobility pattern

Fig. 5.15, 5.16 and table 5.4 show throughput of proposed and compared protocols. Throughput of DBR and EEDBR is almost constant after $100 \mathrm{sec}$. This constant value is due to quick fall in network density. As network density decreases, nodes fail to 
find optimal forwarders for data forwarding due to which throughput attains a constant value. This behaviour is unsuitable for both delay-tolerant and delay-sensitive applications. CoDBR attains high throughput due to cooperatively forwarding the data packets. Throughput of iAMCTD, DEADS-SRC and DEADS-MRC demonstrate the amount of threshold-optimized data obtained from reactive networks. In these protocols, unnecessary data transmission is avoided. In iAMCTD, throughput is high because of the selection of most optimal forwarder. This optimal forwarder is the one that possesses highest residual energy, least depth and depth difference (between source and next-hop node) and best link state at any particular time instant. Hence probability of data packet being corrupted by link impairments are reduced and high throughput is achieved. The proposed model for data routing attains optimal results with linear sink mobility as compared to elliptical sink mobility. Although, in DEADS-SRC and DEADS-MRC, only forwarder node's depth and residual energy are considered as selection parameters represented by eq. 5.11 and eq. 5.15; however, improved results are achieved as compared to iAMCTD due to implementation of cooperative routing. If data received via direct route (source to $D S$ node) is corrupted, it can be received successfully via an alternate route (source to $C C$ to $D S$ node). Along with this, implementation of MRC (diversity combining technique) at $D S$ nodes further improves SNR of received data which results in high throughput. Table 5.4 shows that the highest throughput is achieved DEADS-SRC and DEADS-MRC with linear mobility pattern of MSs. Hence, this particular mathematical model performs the best with linear mobility pattern of MSs.

\begin{tabular}{|l|l|l|l|l|l|l|l|}
\hline Protocol & $\mathbf{5 0} \mathbf{~ s e c}$ & $\mathbf{1 0 0} \mathbf{~ s e c}$ & $\mathbf{1 5 0} \mathbf{~ s e c}$ & $\mathbf{2 0 0} \mathbf{~ s e c}$ & $\mathbf{2 5 0} \mathbf{~ s e c}$ & $\mathbf{3 0 0} \mathbf{~ s e c}$ & $\mathbf{3 5 0} \mathbf{~ s e c}$ \\
\hline DBR & $0.65 \times 10^{4}$ & $0.8 \times 10^{4}$ & $0.8 \times 10^{4}$ & $0.8 \times 10^{4}$ & $0.8 \times 10^{4}$ & $0.8 \times 10^{4}$ & $0.8 \times 10^{4}$ \\
\hline EEDBR & $0.55 \times 10^{4}$ & $0.8 \times 10^{4}$ & $0.8 \times 10^{4}$ & $0.8 \times 10^{4}$ & $0.8 \times 10^{4}$ & $0.8 \times 10^{4}$ & $0.8 \times 10^{4}$ \\
\hline CoDBR & $0.9 \times 10^{4}$ & $1.2 \times 10^{4}$ & $1.25 \times 10^{4}$ & $1.25 \times 10^{4}$ & $1.25 \times 10^{4}$ & $1.25 \times 10^{4}$ & $1.25 \times 10^{4}$ \\
\hline iAMCTD & $0.51 \times 10^{4}$ & $1.28 \times 10^{4}$ & $2.1 \times 10^{4}$ & $2.7 \times 10^{4}$ & $3.18 \times 10^{4}$ & $3.18 \times 10^{4}$ & $3.18 \times 10^{4}$ \\
\hline $\begin{array}{l}\text { DEADS-SRC } \\
\text { (linear) }\end{array}$ & $0.1 \times 10^{4}$ & $1.93 \times 10^{4}$ & $2.53 \times 10^{4}$ & $3.08 \times 10^{4}$ & $3.4 \times 10^{4}$ & $3.4 \times 10^{4}$ & $3.4 \times 10^{4}$ \\
\hline $\begin{array}{l}\text { DEADS-SRC } \\
\text { (Elliptical) }\end{array}$ & $0.8 \times 10^{4}$ & $1.4 \times 10^{4}$ & $1.75 \times 10^{4}$ & $2.07 \times 10^{4}$ & $2.37 \times 10^{4}$ & $2.53 \times 10^{4}$ & $2.57 \times 10^{4}$ \\
\hline $\begin{array}{l}\text { DEADS- } \\
\text { MRC (linear) }\end{array}$ & $0.1 \times 10^{4}$ & $1.93 \times 10^{4}$ & $2.5 \times 10^{4}$ & $2.98 \times 10^{4}$ & $3.15 \times 10^{4}$ & $3.15 \times 10^{4}$ & $3.15 \times 10^{4}$ \\
\hline $\begin{array}{l}\text { DEADS- } \\
\text { MRC (Ellip- } \\
\text { tical) }\end{array}$ & $0.55 \times 10^{4}$ & $1.18 \times 10^{4}$ & $1.67 \times 10^{4}$ & $2.03 \times 10^{4}$ & $2.43 \times 10^{4}$ & $2.75 \times 10^{4}$ & $2.8 \times 10^{4}$ \\
\hline
\end{tabular}

Table 5.4: Network's throughput in bits after equal time intervals (sec) 


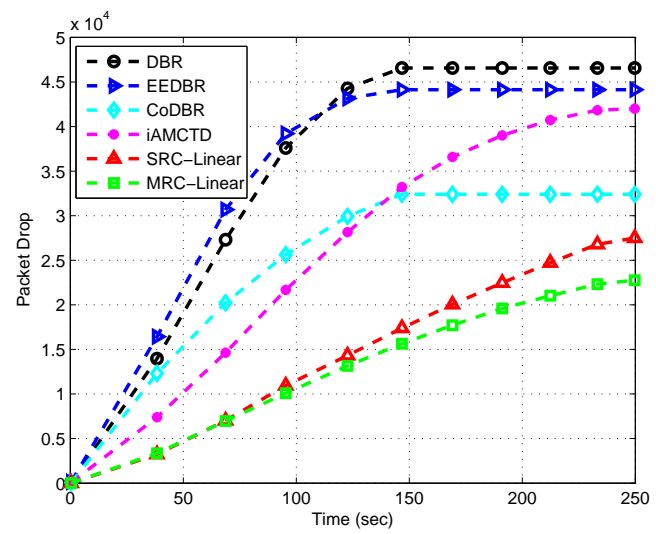

Figure 5.17: Comparison of packet drop with linear sink mobility pattern

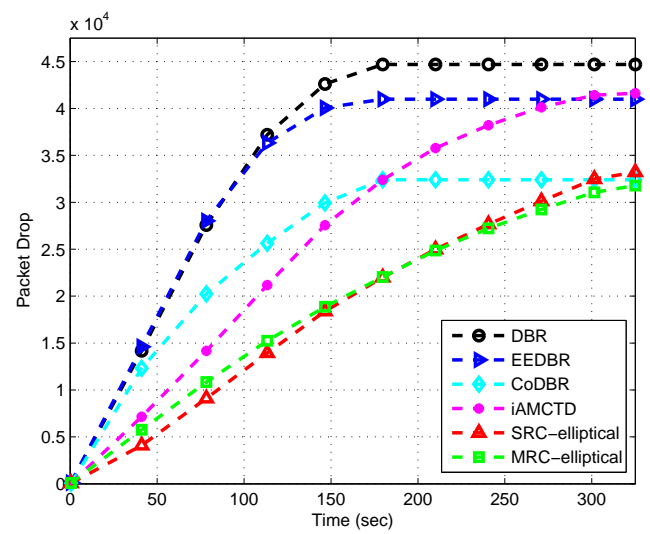

Figure 5.18: Comparison of packet drop with elliptical sink mobility pattern

Fig. 5.17 and 5.18 show packet drop. High packet drop of DBR, EEDBR and iAMCTD is due to the availability of single link with poor link state most of the time. In CoDBR, packet drop is lesser because of cooperative routing. Although, iAMCTD considers link state of potential forwarder node before their selection, even then, the presence of redundant links as well as implementation of MRC (diversity combining technique) gives improved results and makes DEADS-SRC and DEADS-MRC more reliable. In CoDBR, DEADS-SRC and DEADS-MRC, packets are dropped only when no link (direct link to the $D S$ node or via $C C$ to $D S$ node) is available for data forwarding or SNR of combined signal received at $D S$ node (source to $D S$ node, source to $C C$ to $D S$ node) is below an acceptable limit. In CoDBR, multiple transmissions of same data packet are performed before it is received by static sink. Effect of link impairments is high because of these multiple transmissions and as a result packet drop is high. Packet drop is higher in DEADS-SRC and DEADS-MRC with elliptical mobility pattern as compared to linear mobility pattern of MSs. This is because the proposed model is not optimized for elliptical mobility pattern. Whenever the condition(s) specified by $H_{t h}$ and $S_{t h}$ is met, packets are generated by nodes. Sink only accepts those packets that possess an accept- 
able SNR value and drops the remaining packets. In DEADS-SRC and DEADS-MRC with elliptical mobility pattern of MSs, data packets have to traverse multiple hops in order to get received at sink because of which their probability of being erroneous is high which results in packet drop. On the other hand, MSs are available for data reception mostly at one hop distance in linear sink mobility scenario because of which packet drop is reduced. Table 5.5 shows that packet drop rate of DEADS-SRC and DEADS-MRC is lowest with linear mobility pattern of MSs.

\begin{tabular}{|l|l|l|l|l|l|l|l|}
\hline Protocol & $\mathbf{5 0} \mathbf{~ s e c}$ & $\mathbf{1 0 0} \mathbf{~ s e c}$ & $\mathbf{1 5 0} \mathbf{~ s e c}$ & $\mathbf{2 0 0} \mathbf{~ s e c}$ & $\mathbf{2 5 0} \mathbf{~ s e c}$ & $\mathbf{3 0 0} \mathbf{~ s e c}$ & $\mathbf{3 5 0} \mathbf{~ s e c}$ \\
\hline DBR & $2 \times 10^{4}$ & $3.9 \times 10^{4}$ & $4.65 \times 10^{4}$ & $4.65 \times 10^{4}$ & $4.65 \times 10^{4}$ & $4.65 \times 10^{4}$ & $4.65 \times 10^{4}$ \\
\hline EEDBR & $2.25 \times 10^{4}$ & $4 \times 10^{4}$ & $4.4 \times 10^{4}$ & $4.4 \times 10^{4}$ & $4.4 \times 10^{4}$ & $4.4 \times 10^{4}$ & $4.4 \times 10^{4}$ \\
\hline CoDBR & $1.5 \times 10^{4}$ & $2.63 \times 10^{4}$ & $3.25 \times 10^{4}$ & $3.25 \times 10^{4}$ & $3.25 \times 10^{4}$ & $3.25 \times 10^{4}$ & $3.25 \times 10^{4}$ \\
\hline iAMCTD & $1 \times 10^{4}$ & $2.25 \times 10^{4}$ & $3.42 \times 10^{4}$ & $4 \times 10^{4}$ & $4.23 \times 10^{4}$ & $4.23 \times 10^{4}$ & $4.23 \times 10^{4}$ \\
\hline $\begin{array}{l}\text { DEADS-SRC } \\
(\text { linear) }\end{array}$ & $0.5 \times 10^{4}$ & $1.2 \times 10^{4}$ & $1.78 \times 10^{4}$ & $2.38 \times 10^{4}$ & $2.75 \times 10^{4}$ & $2.75 \times 10^{4}$ & $2.75 \times 10^{4}$ \\
\hline $\begin{array}{l}\text { DEADS-SRC } \\
\text { (Elliptical) }\end{array}$ & $0.52 \times 10^{4}$ & $1.23 \times 10^{4}$ & $1.88 \times 10^{4}$ & $2.43 \times 10^{4}$ & $2.86 \times 10^{4}$ & $3.23 \times 10^{4}$ & $3.3 \times 10^{4}$ \\
\hline $\begin{array}{l}\text { DEADS- } \\
\text { MRC (linear) }\end{array}$ & $0.5 \times 10^{4}$ & $1.1 \times 10^{4}$ & $1.52 \times 10^{4}$ & $2 \times 10^{4}$ & $2.28 \times 10^{4}$ & $2.28 \times 10^{4}$ & $2.28 \times 10^{4}$ \\
\hline $\begin{array}{l}\text { DEADS- } \\
\text { MRC (Ellip- } \\
\text { tical) }\end{array}$ & $0.7 \times 10^{4}$ & $1.4 \times 10^{4}$ & $1.9 \times 10^{4}$ & $2.43 \times 10^{4}$ & $2.83 \times 10^{4}$ & $3.11 \times 10^{4}$ & $3.2 \times 10^{4}$ \\
\hline
\end{tabular}

Table 5.5: No. of dropped bits after equal time intervals (sec)

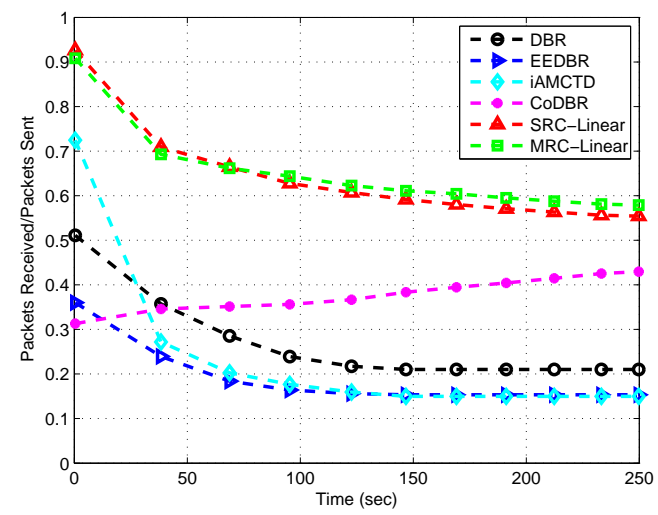

Figure 5.19: Comparison of PAR with linear sink mobility pattern 


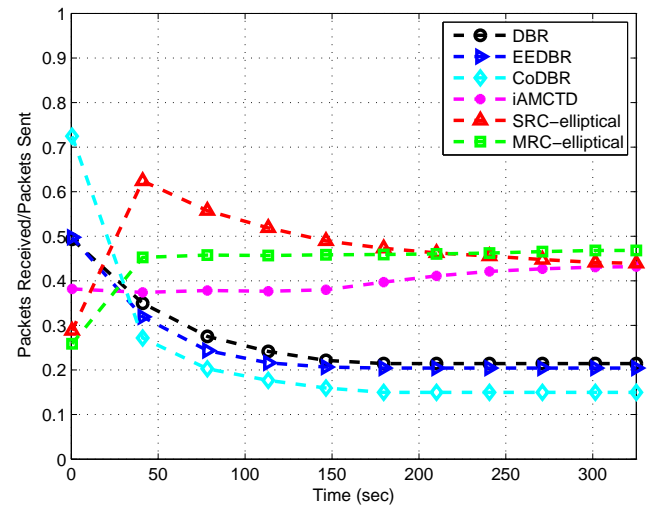

Figure 5.20: Comparison of PAR with elliptical sink mobility pattern

PAR is the ratio of packets successfully received at sink to total number of packets generated by the nodes. PAR is shown in fig. ??. DBR and EEDBR have the lowest PAR as the number of packets received at sink are less due to single link having poor link state most of the time. iAMCTD shows higher PAR than DBR and EEDBR as it selects forwarder nodes on the basis of their respective link's SNR. This results in high throughput and increased PAR. Initially CoDBR attains higher PAR as compared to DBR, EEDBR and iAMCTD which later at decreases as throughput decreases. PAR is higher in DEADS-SRC and DEADS-MRC with linear mobility pattern of MSs as the number of packets received at sink in this scenario is high and packet drop is low.

Table 5.6 shows the improvement attained by our proposed protocol with respect to network lifetime, throughput and packet drop. Zero indicates that both protocols have same value for that specific evaluation metric. Negative value indicates reduced value of a particular metric with respect to compared protocol. Network lifetime of proposed scheme is same as iAMCTD but outperforms DBR and EEDBR. Highest throughput and lowest packet drop is achieved by DEADS-SRC and DEADS-MRC with linear mobility pattern of MSs.

\subsubsection{Performance Tradeoffs Made by DEADS}

DEADS is a reactive routing protocol in which data is transmitted only when certain predefined thresholds are met. Hence it is not suitable for applications where continuous data monitoring and reception are required.

DEADS achieves improved network lifetime by introducing sink mobility in the network. In DEADS, cooperative routing is performed and high throughput is achieved at the cost of increased energy consumption and control overhead. Two to three times more energy consumption is involved when cooperative routing is performed in a network as shown in 


\begin{tabular}{|l|l|l|l|l|l|}
\hline \multirow{2}{*}{ Evaluation metric } & \multirow{2}{*}{ Protocol } & \multicolumn{2}{|c|}{ DEADS-SRC } & \multicolumn{2}{c|}{ DEADS-MRC } \\
\cline { 3 - 6 } & & linear & elliptical & linear & elliptical \\
\hline \multirow{4}{*}{ Network lifetime } & DBR & $40 \%$ & $45 \%$ & $40 \%$ & $45 \%$ \\
\cline { 2 - 6 } & EEDBR & $40 \%$ & $45 \%$ & $40 \%$ & $45 \%$ \\
\cline { 2 - 6 } & CoDBR & $40 \%$ & $45 \%$ & $40 \%$ & $45 \%$ \\
\cline { 2 - 6 } & iAMCTD & 0 & 0 & 0 & 0 \\
\hline \multirow{4}{*}{ Throughput } & DBR & $76 \%$ & $69 \%$ & $75 \%$ & $71 \%$ \\
\cline { 2 - 6 } & EEDBR & $76 \%$ & $69 \%$ & $75 \%$ & $71 \%$ \\
\cline { 2 - 6 } & CoDBR & $63 \%$ & $51 \%$ & $60 \%$ & $55 \%$ \\
\cline { 2 - 6 } & iAMCTD & $6.4 \%$ & $-23 \%$ & $-1 \%$ & $-13 \%$ \\
\hline \multirow{3}{*}{ Packet drop } & DBR & $-41 \%$ & $-29 \%$ & $-51 \%$ & $-31 \%$ \\
\cline { 2 - 6 } & EEDBR & $-37.5 \%$ & $-25 \%$ & $-48 \%$ & $-27 \%$ \\
\cline { 2 - 6 } & CoDBR & $-15 \%$ & $1.5 \%$ & $-30 \%$ & $-1.5 \%$ \\
\cline { 2 - 6 } & iAMCTD & $-35 \%$ & -22 & $-46 \%$ & $-24 \%$ \\
\hline
\end{tabular}

Table 5.6: Performance comparison of DEADS-SRC and DEADS-MRC with counterpart techniques

fig. 5.13 and 5.14. Along with this, control packet exchange for selection of cooperative partner nodes increases control overhead.

An optimized value of $d_{t h}$ in accordance to eq. 5.4 is attained at the cost of control packet exchange. Nodes require their one-hop neighbor information in order to select their $d_{t h}$.

There is a tradeoff between networks stability period and data reliability. Stability period is compromised in order to attain reliable data transfer as shown in fig. 5.11 and 5.12. Data reliability refers to the authenticity of received data and it is affected by the damage caused by link impairments. More is the effect of link impairments on the received data, lesser is its reliability and more are the chances of it being dropped by receiving node and vice verse. Where, the link state varies from node to node. Higher energy consumption causes nodes to die at the start of the network compromising stability period, however, because of transmission via alternate paths data reliability is achieved.

Reliable data transmission results in high throughput, as shown in fig. 5.15 and 5.16 because chances of successful data reception at sink are increased. Hence, there is a tradeoff between throughput and energy consumption.

Reliable data transfer is attained at the cost of increased processing overhead (in terms of MRC and AF) and increased delay. Data is transmitted from source to relay and destination node. Relay node after performing $\mathrm{AF}$ at the received data forwards it to destination node. Destination node performs MRC at the received copies, selects appropriate relay and destination node and then forwards the data. In all this processing certain overhead is involved. Delay is also introduced because of which the proposed protocol is not suitable for delay sensitive applications. 
DEADS achieves reduced packet drop as shown in fig. 5.17 and 5.18 at the cost of multiple transmissions. Greater number of transmissions increase reliability at the cost of extra energy consumption. Tradeoffs are shown in table 5.7.

\begin{tabular}{|c|c|c|c|}
\hline DEADS & $\begin{array}{l}\text { Modification over } \\
\text { existing protocols }\end{array}$ & Advances achieved & Price to pay \\
\hline $\begin{array}{l}\text { DEADS- } \\
\text { SRC }\end{array}$ & $\begin{array}{l}\text { Single relay cooperative } \\
\text { routing along with sink } \\
\text { mobility }\end{array}$ & $\begin{array}{l}\text { High throughput (fig. } \\
5.15 \text { and } 5.16 \text { ), reduced } \\
\text { packet drop (fig. } 5.17 \\
\text { and } 5.18 \text { ), optimized } d_{t h} \\
\text { (rule 4) }\end{array}$ & $\begin{array}{l}\text { High energy consump- } \\
\text { tion (fig. } 5.13 \text { and } 5.14 \text { ), } \\
\text { stability period compro- } \\
\text { mised (fig. } 5.11 \text { and } \\
5.12 \text { ), control and pro- } \\
\text { cessing overhead }\end{array}$ \\
\hline $\begin{array}{l}\text { DEADS- } \\
\text { MRC }\end{array}$ & $\begin{array}{l}\text { Multiple relay coopera- } \\
\text { tive routing along with } \\
\text { sink mobility }\end{array}$ & $\begin{array}{l}\text { High throughput (fig. } \\
5.15 \text { and } 5.16 \text { ), reduced } \\
\text { packet drop (fig. } 5.17 \\
\text { and } 5.18 \text { ), optimized } d_{t h} \\
\text { (rule } 4 \text { ) }\end{array}$ & $\begin{array}{l}\text { High energy consump- } \\
\text { tion (fig. } 5.13 \text { and } 5.14 \text { ), } \\
\text { stability period compro- } \\
\text { mised (fig. } 5.11 \text { and } \\
5.12 \text { ), control and pro- } \\
\text { cessing overhead }\end{array}$ \\
\hline
\end{tabular}

Table 5.7: Performance tradeoffs made by routing protocols 
Chapter 6

Conclusion 
In this thesis, we proposed a DS based cooperative routing algorithm along with sink mobility for enhancing the performance of UWSNs. The proposed algorithm is devised by taking into account depth and residual energy as routing metrics. It operates in three different phases, namely; neighbor selection phase, DS and CC set formation phase and threshold based data sensing and routing phase. During the neighbor selection phase, source nodes select their $d_{t h}$, find neighbors, and attain their depth and residual energy information. During the DS and CC set formation phase, source nodes utilize the information attained in previous phase and select their respective DS and CC nodes for cooperative routing. In threshold based data sensing and routing phase, source nodes sense data on the basis of the pre-defined threshold and forward it to their cooperative partner nodes. Along with this algorithm, two different criteria for cooperative partner node selection are also implemented and compared in this work. A links SNR gives better approximation of the link because of which SNR based criterion results in the selection of optimal forwarder nodes. We implemented our proposed work in MATLAB and evaluated its performance by comparing it with DBR, EEDBR, and iAMCTD with respect to network lifetime, throughput, PAR and energy consumption. Based on the comprehensive simulation, we observe that our work contributes to the performance improvement in terms of network lifetime as compared to DBR and EEDBR. High throughput, reduced packet drop and improved PAR is achieved as compared to all three depth-based routing protocols i.e. DBR, EEDBR and iAMCTD which makes the proposed work most reliable of all. 
Chapter 7

References 
[1] M. Al-Bzoor, Y. Zhu, J. Liu, R. Ammar, J.-H. Cui, and S. Rajasekaran, "An adaptive surface sink redeployment strategy for underwater sensor networks," in Computers and Communications (ISCC), 2013 IEEE Symposium on, pp. 000801-000806, IEEE, 2013.

[2] S. I. Aldharrab, Cooperative Communication over Underwater Acoustic Channels. $\mathrm{PhD}$ thesis, University of Waterloo, 2013.

[3] M. J. Jannati, A. D. Aref, and V. T. Vakily, "Implementation of cooperative virtual miso communication in underwater acoustic wireless sensor networks," International Journal of Computer Science Issues (IJCSI), vol. 8, no. 4, 2011.

[4] Y. Luo, L. Pu, Z. Peng, Z. Zhou, and J.-H. Cui, "Ct-mac: a mac protocol for underwater mimo based network uplink communications," in Proceedings of the Seventh ACM International Conference on Underwater Networks and Systems, p. 23, ACM, 2012.

[5] P. Wang, L. Zhang, and V. O. Li, "Asynchronous cooperative transmission for threedimensional underwater acoustic networks," IET Communications, vol. 7, no. 4, pp. 286-294, 2013.

[6] C. Gao, Z. Liu, B. Cao, and L. Mu, "Relay selection scheme based on propagation delay for cooperative underwater acoustic network," in Wireless Communications 86 Signal Processing (WCSP), 2013 International Conference on, pp. 1-6, IEEE, 2013.

[7] Y. Luo, L. Pu, Z. Peng, Z. Zhou, J.-H. Cui, and Z. Zhang, "Effective relay selection for underwater cooperative acoustic networks," in Mobile Ad-Hoc and Sensor Systems (MASS), 2013 IEEE 10th International Conference on, pp. 104-112, IEEE, 2013.

[8] A. Umar, M. Akbar, S. Ahmed, N. Javaid, Z. A. Khan, and U. Qasim, "Underwater wireless sensor networks performance enhancement with cooperative routing and sink mobility," in Broadband and Wireless Computing, Communication and Applications (BWCCA), 2014 Ninth International Conference on. 
[9] H. Nasir, N. Javaid, Z. A. Khan, S. Mansoor, U. Qasim, and M. Sher, "Codbr: Cooperative depth based routing for underwater wireless sensor networks," in Broadband and Wireless Computing, Communication and Applications (BWCCA), 2014 Ninth International Conference on.

[10] R. Su, R. Venkatesan, and C. Li, "A new node coordination scheme for data gathering in underwater acoustic sensor networks using autonomous underwater vehicle," in Wireless Communications and Networking Conference (WCNC), 2013 IEEE, pp. 4370-4374, IEEE, 2013.

[11] J. Wang, D. Li, M. Zhou, and D. Ghosal, "Data collection with multiple mobile actors in underwater sensor networks," in Distributed Computing Systems Workshops, 2008. ICDCS'08. 28th International Conference on, pp. 216-221, IEEE, 2008.

[12] S. Shen, A. Zhan, P. Yang, and G. Chen, "Exploiting sink mobility to maximize lifetime in 3d underwater sensor networks," in Communications (ICC), 2010 IEEE International Conference on, pp. 1-5, IEEE, 2010.

[13] J. Luo, J. Panchard, M. Piórkowski, M. Grossglauser, and J.-P. Hubaux, "Mobiroute: Routing towards a mobile sink for improving lifetime in sensor networks," in Distributed Computing in Sensor Systems, pp. 480-497, Springer, 2006.

[14] S. Yoon, A. K. Azad, H. Oh, and S. Kim, "Aurp: An auv-aided underwater routing protocol for underwater acoustic sensor networks," Sensors, vol. 12, no. 2, pp. $1827-$ $1845,2012$.

[15] A. Ahmad, A. Wahid, and D. Kim, "Aeerp: Auv aided energy efficient routing protocol for underwater acoustic sensor network," in Proceedings of the 8th ACM workshop on Performance monitoring and measurement of heterogeneous wireless and wired networks, pp. 53-60, ACM, 2013.

[16] H. Yan, Z. J. Shi, and J.-H. Cui, "Dbr: Depth-based routing for underwater sensor networks," in Adhoc and Sensor Networks, pp. 72-86, Springer, 2008.

[17] A. Wahid, S. Lee, H.-J. Jeong, and D. Kim, "Eedbr: Energy-efficient depth-based routing protocol for underwater wireless sensor networks," in Advanced Computer Science and Information Technology, pp. 223-234, Springer, 2012.

[18] N. Javaid, M. Jafri, Z. Khan, U. Qasim, T. Alghamdi, and M. Ali, "iamctd: improved adaptive mobility of courier nodes in threshold-optimized dbr protocol for underwater wireless sensor networks,"

[19] L. Freitag, M. Grund, S. Singh, J. Partan, P. Koski, and K. Ball, "The whoi micromodem: an acoustic communications and navigation system for multiple platforms," in OCEANS, 2005. Proceedings of MTS/IEEE, pp. 1086-1092, IEEE, 2005. 
[20] M. Stojanovic, "On the relationship between capacity and distance in an underwater acoustic communication channel," ACM SIGMOBILE Mobile Computing and Communications Review, vol. 11, no. 4, pp. 34-43, 2007.

[21] R. J. Urick, Principles of underwater sound for engineers. Tata McGraw-Hill Education, 1967.

[22] A. F. Harris III and M. Zorzi, "Modeling the underwater acoustic channel in ns2," in Proceedings of the 2nd international conference on Performance evaluation methodologies and tools, p. 18, ICST (Institute for Computer Sciences, Social-Informatics and Telecommunications Engineering), 2007.

[23] J. Heidemann, M. Stojanovic, and M. Zorzi, "Underwater sensor networks: applications, advances and challenges," Philosophical Transactions of the Royal Society A: Mathematical, Physical and Engineering Sciences, vol. 370, no. 1958, pp. 158-175, 2012.

[24] M. Ayaz, A. Abdullah, I. Faye, and Y. Batira, "An efficient dynamic addressing based routing protocol for underwater wireless sensor networks," Computer Communications, vol. 35, no. 4, pp. 475-486, 2012.

[25] J. Wills, W. Ye, and J. Heidemann, "Low-power acoustic modem for dense underwater sensor networks," in Proceedings of the 1st ACM international workshop on Underwater networks, pp. 79-85, ACM, 2006.

[26] K. El-Darymli, "Amplify-and-forward cooperative relaying for a linear wireless sensor network," in Systems Man and Cybernetics (SMC), 2010 IEEE International Conference on, pp. 106-112, IEEE, 2010. 